\title{
FOURIER TRANSFORMS OF NONCOMMUTATIVE ANALOGUES OF VECTOR MEASURES AND BIMEASURES WITH APPLICATIONS TO STOCHASTIC PROCESSES
}

\author{
KARI YLINEN
}

\section{Introduction}

I. Kluvánek [14] has studied Fourier-Stieltjes transforms of Banach space valued regular Borel vector measures on a locally compact Abelian topological group $\Gamma$. Let us think of $\Gamma$ as the dual group of its own dual group $G$. If $E$ is a (complex) Banach space, the Fourier-Stieltjes transform of a regular $E$-valued Borel vector measure $m$ on $\Gamma$ is the function $\varphi: G \rightarrow E$ defined by

$$
\varphi(s)=\int_{\Gamma} \overline{(s, \gamma)} d m(\gamma), \quad s \in G .
$$

The principal results in [14] concern the problem of characterizing those $E$-valued functions on $G$ which arise as Fourier-Stieltjes transforms of $E$-valued regular Borel vector measures on $\Gamma$. Kluvánek proved, generalizing two classical theorems of W. F. Eberlein [7] on Fourier-Stieltjes transforms of scalar valued measures, that a function $\varphi: G \rightarrow E$ is the FourierStieltjes transform of such a vector measure if and only if $\varphi$ is bounded and weakly continuous and the integrals $\int_{G} \varphi d \mu$ form a relatively weakly compact subset of $E$, when $\mu$ ranges over the bounded absolutely continuous (resp. finitely supported) regular complex Borel measures on $G$ whose Fourier-Stieltjes transforms are bounded by 1 (see Theorems 2 and 3 in [14]). These conditions even imply that $\varphi$ is strongly continuous [14, p. 269]. The present article originated in an attempt to extend the results quoted above to the case of a not necessarily Abelian locally compact group G. A complete generalization is contained in Theorems 3.2 and 3.5. Instead of entering a detailed discussion in this introductory section we only mention that one replaces vector measures by weakly compact linear 
operators from $C^{*}(G)$ to $E$, where $C^{*}(G)$, the so-called group $C^{*}$ algebra of $G$, can be regarded as a generalization of $C_{0}(\Gamma)$ (see Sections 2 and 5). This approach is motivated by the fact that the $E$-valued regular Borel vector measures on $\Gamma$ are known to be via integration in a bijective correspondence with the weakly compact operators from $C_{0}(\Gamma)$ to $E$.

The second main purpose of this paper is to define and characterize the Fourier transforms of bounded bilinear forms on the Cartesian product of the group $C^{*}$-algebras of two locally compact groups $G_{1}$ and $G_{2}$. This is done in Section 4. Our definition depends on the extending of the bilinear form to $C^{*}\left(G_{1}\right) * * \times C^{*}\left(G_{2}\right) * *$. That this extension can be effected in a satisfactory way is guaranteed by a result of $\mathrm{C}$. A. Akemann [1], which says that any bounded linear operator from a $C^{*}$-algebra to the predual of a von Neumann algebra is weakly compact. The Fourier transform of a bounded bilinear form on $C^{*}\left(G_{1}\right) \times C^{*}\left(G_{2}\right)$ is a bounded continuous function on $G_{1} \times G_{2}$. The principal result of Section 4 is Theorem 4.7 , which singles out, in a manner analogous to the two theorems of Eberlein referred to above, those functions on $G_{1} \times G_{2}$ which arise as such Fourier transforms. Theorems 4.10 and 4.12 deal with positive-definiteness properties of bounded bilinear forms.

Our study of the Fourier transforms of bounded bilinear forms on $C^{*}\left(G_{1}\right) \times C^{*}\left(G_{2}\right)$ is largely motivated by applications to the theory of stochastic processes indexed by a general locally compact group. This theme is discussed in Section 6. There our main concern is the generalization of the classical spectral representation theory. Our approach resembles that of H. Niemi [17]. For example, we extend to the noncommutative case the notion of the covariance bimeasure of a stochastic measure considered in [17]. This provides the essential link between Sections 4 and 6 .

We use the same basic techniques of noncommutative harmonic analysis as e.g. P. Eymard [8]. The general framework will be explained in the next section. In the commutative case, the notions reviewed there reduce to standard concepts of harmonic analysis on locally compact Abelian groups. The implications of this reduction in the situations considered in Sections 3 and 4 will be discussed in Section 5. This is done mainly for the purpose of illustration; of course the commutative theory could be reached by a more direct route. At certain points, however, the application of $C^{*}$ algebra techniques (for example, the use of Kaplansky's density theorem in the proofs of Theorems 3.5 and 4.10) sheds new light even on the commutative case. 


\section{Preliminaries}

The notation, terminology and results set out in this section will be used throughout the paper, usually without explicit reference. The scalar field will always be the complex field $\mathbf{C}$. Let $E$ be a Banach space. Then $E^{*}$ denotes its (topological) dual, $\sigma\left(E, E^{*}\right)$ is by definition the weak topology on $E$, and $\sigma\left(E^{*}, E\right)$ is the weak* topology on $E^{*}$. The value of $f \in E^{*}$ at $x \in E$ is denoted interchangeably by $\langle f, x\rangle$ or $\langle x, f\rangle$. The basic theory of Banach spaces and topological vector spaces is assumed to be known. For example, we frequently use the fact that if two vector spaces $E$ and $F$ are in duality, then any linear subspace $F_{1} \subset F$ which suffices to separate the points of $E$ is $\sigma(F, E)$-dense in $F$ (see e.g. [22, p. 125]). For the theory of $C^{*}$-algebras, von Neumann algebras, and group representations we refer to [4], [5] and [21]. A von Neumann algebra may be defined as a $C^{*}$-algebra $M$ which is the dual of a (necessarily unique) Banach space $M_{*}$, called the predual of $M$. In any von Neumann algebra $M$, involution is $\sigma\left(M, M_{*}\right)$-continuous and multiplication separately $\sigma\left(M, M_{*}\right)$-continuous (see e.g. [21, p. 18]). The bidual of any $C^{*}$-algebra $A$ is a $C^{*}$-algebra, which can be identified with the so-called enveloping von Neumann algebra of $A$ [5, p. 236]. We shall always consider $A^{* *}$ to be equipped with this structure. The canonical embedding of $A$ into $A^{* *}$ is then a *-homomorphism. Kaplansky's density theorem will be used in the following form: if $A$ is a $\sigma\left(M, M_{*}\right)$-dense *-subalgebra of the von Neumann algebra $M$, then the unit ball of $A$ is $\sigma\left(M, M_{*}\right)$-dense in the unit ball of $M$ (see [4, pp. 41, 43], [21, p. 22]).

If $X$ is a locally compact Hausdorff space, $C_{0}(X)$ denotes the $C^{*}$. algebra of all continuous complex functions on $X$ vanishing at infinity, equipped with the supremum norm. As usual, $C_{0}(X) *$ is identified with $M(X)$, the space of bounded regular complex Borel measures on $X$. The subspace of $M(X)$ consisting of all finite linear combinations of the Dirac measures $\delta_{s}, s \in X$, is denoted by $M_{d d}(X)$. The basic theory of measure and integration that we use can be found e.g. in [12].

Let $G$ be a locally compact (Hausdorff) topological group with a fixed left Haar measure $d s$. We shall regard the Banach space $L^{1}(G)$ consisting of the (equivalence classes of) complex Borel functions on $G$ integrable with respect to $d s$ as a closed subspace of $M(G)$ by identifying $g \in L^{1}(G)$ with the measure $\mu$ for which $d \mu=g d s$. Equipped with the convolution product and a natural involution $\mu \mapsto \mu^{*}$ (see e.g. [5, p. 252], [12, p. 299]), $M(G)$ is a Banach *-algebra having $L^{1}(G)$ as a closed *-ideal.

Each continuous unitary representation $\pi$ of $G$ on a Hilbert space $H_{\pi}$ determines a *-representation (also denoted by $\pi$ ) of $M(G)$ on $H_{\pi}$ satisfying 


$$
(\pi(\mu) \xi \mid \eta)=\int_{G}(\pi(s) \xi \mid \eta) d \mu(s) \text { for } \mu \in M(G), \xi, \eta \in H_{\pi}
$$

(the inner product of a Hilbert space is in this paper always denoted by $(\cdot \mid \cdot))$. The restriction of $\pi$ to $L^{1}(G)$ is essential ("non dégénérée" in the terminology of [5]), and each essential *-representation of $L^{1}(G)$ is obtained in this way from a unique continuous unitary representation of $G[5, \mathrm{p}$. 253]. If $\mu \in M(G)$, we let $\|\mu\|^{\prime}$ denote the supremum of the operator norms $\|\pi(\mu)\|$ where $\pi$ ranges over all continuous unitary representations of $G$. Then $\|\mu\|^{\prime} \leq\|\mu\|\left[5\right.$, p. 7]. The completion of $L^{1}(G)$ with respect to the norm $\|\cdot\|^{\prime}$ is a $C^{*}$-algebra, which is denoted by $C^{*}(G)$ and called the group $C^{*}$-algebra of $G$ (see $\left[5\right.$, p. 271]). Let $\omega: C^{*}(G) \rightarrow L\left(H_{\omega}\right)$ be the universal representation of $C^{*}(G)$ [5, p. 43]. If $\tau: L^{1}(G) \rightarrow C^{*}(G)$ is the inclusion map, $\omega \circ \tau$ is an essential *-representation of $L^{1}(G)$. We denote $\omega \circ \tau$ and the corresponding continuous unitary representation of $G$ on the Hilbert space $H_{\omega}$ simply with $\omega$. The von Neumann algebra $A$ generated in $L\left(H_{\omega}\right)$ by $\omega\left(C^{*}(G)\right)$ is just the enveloping von Neumann algebra of $C^{*}(G)$ mentioned earlier, and it can be identified with the bidual of $C^{*}(G)$ as described e.g. in [5, p. 236]. In this identification the canonical embedding $x: C^{*}(G) \rightarrow C^{*}(G) * *$ corresponds to $\omega: C^{*}(G) \rightarrow L\left(H_{\omega}\right)$, which shows that $\omega(\mu)=\varkappa(\mu)$ whenever $\mu \in L^{1}(G) \subset C^{*}(G)$. Since $\omega(\mu) \in A$ for all $\mu \in M(G) \quad[8$, p. 193], $\omega$ will usually be regarded as a mapping from $M(G)$ into $C^{*}(G) * *$ (although we continue to let $\omega$ also denote its restriction to $G$ which is considered canonically embedded in $M(G)$ ) .

For each $\mu \in M(G),\|\mu\|^{\wedge}$ will denote the supremum of the operator norms $\|\pi(\mu)\|$ where $\pi$ ranges over the continuous (topologically) irreducible unitary representations of $G$. We have $\|\mu\|^{\wedge}=\|\mu\|^{\prime}=$ $\|\omega(\mu)\|$ if $\mu \in L^{1}(G) \quad[5$, pp. 40, 254-255]. From Lemma 1.23 in [8, p. 189] it follows therefore that $\|\mu\|^{\wedge}=\|\mu\|^{\prime}=\|\omega(\mu)\|$ for all $\mu \in M(G)$. In particular, the *-algebra homomorphism $\omega: M(G) \rightarrow C^{*}(G) * *$ is injective, because $\|\mu\|^{\prime}>0$ for each nonzero $\mu \in M(G)$ [12, p. 342].

The set of linear combinations of all continuous positive-definite [5, p. 255] complex functions on $G$ will be denoted by $B(G)$. Every function in $B(G)$ is bounded [5, p. 256]. For future reference we collect into two lemmas some properties of $B(G)$ from [8].

$\mathrm{L}$ e $\mathrm{m} \mathrm{m}$ a 2.1. Let $f: G \rightarrow \mathbf{C}$ be bounded and continuous. Then

$$
\begin{aligned}
& \sup \left\{\left|\int f d \mu\right| \mid \mu \in L^{1}(G),\|\mu\|^{\prime} \leq 1\right\} \\
= & \sup \left\{\left|\int f d \mu\right| \mid \mu \in M_{d d}(G),\|\mu\|^{\prime} \leq 1\right\} .
\end{aligned}
$$

This quantity is finite if and only if $f \in B(G)$. 
Proof. Let $a$ (resp. $b$ ) denote the left (resp. right) side of (1). Then $a<\infty$ if and only if $f \in B(G)$ [8, p. 191]. If $f \in B(G)$, then $b<\infty$ and $a=b$ (see (2.13) in [8, p. 195]). Thus it suffices to show that $f \in B(G)$ if $b<\infty$. The proof of this implication will be reduced to $(2.24)$ in [8, p. 202]. Since $\left\|\mu_{n}-\mu\right\|^{\prime} \rightarrow 0$ if $\mu_{n} \rightarrow \mu$ in $M(G)=C_{0}(G)^{*}$, we have

$$
b=\sup \left\{\left|\int f d \mu\right| \mid \mu \in M_{d}(G),\|\mu\|^{\prime} \leq 1\right\},
$$

where $M_{d}(G)$ is the norm closure of $M_{d d}(G)$ in $M(G)$. Let $G_{d}$ denote $G$ equipped with the discrete topology. If $\|\mu\|_{d}^{\prime}$ for $\mu \in L^{1}\left(G_{d}\right)=M\left(G_{d}\right)=$ $M_{d}(G)$ is the supremum of $\|\pi(\mu)\|$ over all unitary representations $\pi$ of $G_{d}$, then clearly $\|\mu\|_{d}^{\prime} \geq\|\mu\|^{\prime}$, so that

$$
\begin{array}{r}
\sup \left\{\left|\int f d \mu\right| \mid \mu \in L^{1}\left(G_{d}\right),\|\mu\|_{d}^{\prime} \leq 1\right\} \\
\leq \sup \left\{\left|\int f d \mu\right| \mid \mu \in M_{d}(G),\|\mu\|^{\prime} \leq 1\right\}=b .
\end{array}
$$

Therefore, if $b<\infty$, then $f \in B\left(G_{d}\right)$ [8, p. 191], and so using the continuity of $f$ on $G$ we get $f \in B(G)$ by $(2.24)$ in [8, p. 202].

There is a bijection $T: B(G) \rightarrow C^{*}(G)^{*}$ which maps the set of the positive-definite functions in $B(G)$ onto the set of the positive linear functionals on $C^{*}(G)$ and satisfies

$$
\langle T f, \mu\rangle=\int f d \mu \text { for all } f \in B(G), \mu \in L^{1}(G) \subset C^{*}(G)
$$

[8, p. 192]. Clearly, $\|T f\|$ is the same as either side of (1) in Lemma 2.1.

$\mathrm{L}$ e $\mathrm{m}$ a 2.2. If $f \in B(G)$, then $\langle\omega(\mu), T f\rangle=\int f d \mu$ for all $\mu \in M(G)$.

Proof. See [8, p. 193].

This lemma shows, in particular, that $\langle\omega(s), T f\rangle=f(s)$ for all $f \in B(G), \quad s \in G$. It follows that if $g \in C^{*}(G)^{*}$, then the function $s \mapsto\langle\omega(s), g\rangle$ on $G$ belongs to $B(G)$, and that $\omega: G \rightarrow C^{*}(G)^{* *}$ is weak* continuous. Furthermore, $\omega\left(M_{d d}(G)\right)$ suffices to separate the points of $C^{*}(G)^{*}$, so that it is weak* dense in $C^{*}(G)^{* *}$.

Whenever we consider two locally compact groups $G_{1}$ and $G_{2}$, the notation introduced in this section will be adapted to that situation without further comments. Thus, for $j=1,2$, the symbols $\omega_{j}, T_{j}$ etc. will have their obvious meanings.

As mentioned in the introduction, the concepts discussed above have simple interpretations in case $G$ is Abelian. By Bochner's theorem [20, p. 19], $\mathrm{B}(\mathrm{G})$ is the set of the Fourier-Stieltjes transforms of the measures in 
$M(\Gamma)$, where $\Gamma$ is the dual group of $G$. Since the set of the (equivalence classes of) continuous irreducible unitary representations of $G$ can be identified with $\Gamma$, the number $\|\mu\|^{\wedge}\left(=\|\mu\|^{\prime}=\|\omega(\mu)\|\right)$ is for every $\mu \in M(G)$ equal to $\|\hat{\mu}\|_{\infty}$, where $\hat{\mu}$ is the Fourier-Stieltjes transform of $\mu$. From well-known properties of the Fourier transformation on $L^{1}(G)$ it follows that $C^{*}(G)$ is isometrically *-isomorphic to $C_{0}(T)$. We shall return to the Abelian case in Section 5.

\section{Fourier transforms of weakly compact operators from $C^{*}(G)$ to a Banach space}

Throughout this section, $G$ is an arbitrary locally compact group, and $E$ is a Banach space. The Fourier transform of a weakly compact operator $\Phi: C^{*}(G) \rightarrow E$ defined here generalizes the notion of the Fourier-Stieltjes transform of an $E$-valued regular Borel vector measure on the dual group of a locally compact Abelian group. We postpone the explanation of this statement to Section 5, and consider only the general situation in this section.

In the next definition, and elsewhere, we shall regard the second adjoint $\Phi^{* *}$ of a weakly compact operator $\Phi: C^{*}(G) \rightarrow E$ as a mapping from $C^{*}(G)^{* *}$ into $E$, because $\Phi^{* *}\left(C^{*}(G)^{* *}\right) \subset E$ ( $\left.\subset E^{* *}\right)[6$, p. 482].

$\mathrm{D}$ efin ition 3.1. If $\Phi: C^{*}(G) \rightarrow E$ is a weakly compact operator, the function $\hat{\Phi}: G \rightarrow E$ defined by $\hat{\Phi}(s)=\Phi^{* *}(\omega(s)), s \in G$, is called the Fourier transform of $\Phi$.

The following theorem exhibits some basic properties of the Fourier transform. Observe that since $\omega: G \rightarrow C^{*}(G)^{* *}$ is weak* continuous and $\Phi^{* *}$ is continuous from $\sigma\left(C^{*}(G)^{* *}, C^{*}(G)^{*}\right)$ to $\sigma\left(E, E^{*}\right)$, it is obvious that $\hat{\Phi}$ is weakly continuous. The proof of its norm continuity, however, turns out to require a considerably more refined method.

Th e o r e m 3.2. If $\Phi_{1}: C^{*}(G) \rightarrow E$ and $\Phi_{2}: C^{*}(G) \rightarrow E$ are weakly compact operators such that $\hat{\Phi}_{1}=\hat{\Phi}_{2}$, then $\Phi_{1}=\Phi_{2}$. The Fourier transform of any weakly compact operator $\Phi: C^{*}(G) \rightarrow E$ is strongly continuous, and $\|\hat{\Phi}(s)\| \leq\|\Phi\|$ for all $s \in G$.

Proof. If $\hat{\Phi}_{1}=\hat{\Phi}_{2}$, then $\Phi_{1}=\Phi_{2}$ by virtue of the fact that $\left\langle f \circ \Phi_{1}, \omega(s)\right\rangle=\left\langle f \circ \Phi_{2}, \omega(s)\right\rangle$ for all $s \in G, f \in E^{*}$, and $\omega(G)$ separates the points of $C^{*}(G)^{*}$. We have $\|\hat{\Phi}(s)\| \leq\left\|\Phi^{* *}\right\|\|\omega(s)\|=\|\Phi\|$ if $s \in G$. Our proof of the norm continuity of $\hat{\Phi}$ is based on a characterization of the relatively weakly compact subsets of the predual of a von Neumann algebra due to C. A. Akemann [1]. Fix $s \in G$, and let $\left(s_{i}\right)_{i \in I}$ be an arbitrary net in $G$ converging to $s$. We intend to prove that $\hat{\Phi}\left(s_{i}\right) \rightarrow \hat{\Phi}(s)$ in 
norm. Let 1 denote the identity element of $C^{*}(G)^{* *}$. As $\omega(s)$ and $\omega\left(s_{i}\right)$ for all $i \in I$ are unitary, we have

$$
\begin{aligned}
& \lim _{i}\left(\omega\left(s_{i}\right)-\omega(s)\right) *\left(\omega\left(s_{i}\right)-\omega(s)\right) \\
& =1-\lim _{i} \omega\left(s_{i}\right) * \omega(s)-\lim _{i} \omega(s)^{*} \omega\left(s_{\imath}\right)+1=0
\end{aligned}
$$

with respect to $\sigma\left(C^{*}(G)^{* *}, C^{*}(G)^{*}\right)$ by the weak* continuity of $\omega$ combined with the fact that involution is weak* continuous and multiplication separately weak* continuous in $C^{*}(G)^{* *}$. Similarly,

$$
\left(\omega\left(s_{i}\right)-\omega(s)\right)\left(\omega\left(s_{i}\right)-\omega(s)\right)^{*} \rightarrow 0 \text { for } \sigma\left(C^{*}(G) * *, C^{*}(G)^{*}\right) .
$$

By Gantmacher's theorem [6, p. 485], $\left\{\Phi^{*} f \mid f \in E^{*},\|f\| \leq 1\right\}$ is a relatively weakly compact subset of $C^{*}(G)^{*}$. Let $\varepsilon>0$ be given. Theorem II.3 in [1, p. 289] yields a positive functional $g \in C^{*}(G)^{*}$ and $\delta>0$ such that $\left|\left\langle u, \Phi^{*} f\right\rangle\right|<\varepsilon / 2$ for all $f \in E^{*}$ with $\|f\| \leq 1$, whenever $u \in C^{*}(G)^{* *},\|u\| \leq 1$ and $g\left(u^{*} u+u u^{*}\right)<\delta$. Choosing $u_{i}=2^{-1}\left[\omega\left(s_{i}\right)-\omega(s)\right]$ for $i \in I$, we have

$$
\lim _{i} g\left(u_{i}^{*} u_{i}+u_{i} u_{i}^{*}\right)=0 .
$$

Thus there is $i_{0} \in I$ such that $i \geq i_{0}$ implies $\left|\left\langle\Phi^{* *}\left(\omega\left(s_{i}\right)-\omega(s)\right), f\right\rangle\right|=$ $\left|\left\langle 2 u_{i}, \Phi^{*} f\right\rangle\right|<\varepsilon$ for all $f \in E^{*},\|f\| \leq 1$, i.e. $\left\|\hat{\Phi}\left(s_{i}\right)-\hat{\Phi}(s)\right\|<\varepsilon$.

The or e m 3.3. A function $\varphi: G \rightarrow E$ is the Fourier transform of some weakly compact operator $\Phi: C^{*}(G) \rightarrow E$ if and only if $f \circ \varphi \in B(G)$ for all $f \in E^{*}$ and the set $\left\{T(f \circ \varphi) \mid f \in E^{*},\|f\| \leq 1\right\}$ is relatively weakly compact in $C^{*}(G)^{*}$. If these conditions hold, then $\|\Phi\|=$ $\sup \left\{\|T(f \circ \varphi)\| \mid f \in E^{*},\|f\| \leq 1\right\}$.

Proof. Suppose first that $\varphi=\hat{\Phi}$ for a weakly compact operator $\Phi: C^{*}(G) \rightarrow E$. If $f \in E^{*}$, then $\Phi^{*} f \in C^{*}(G)^{*}$, and so the function $s \mapsto\left\langle\Phi^{*} f, \omega(s)\right\rangle=\left\langle f, \Phi^{* *}(\omega(s))\right\rangle=f(\varphi(s))$ on $G$ belongs to $B(G)$. By Gantmacher's theorem [6, p. 485], $\Phi^{*}: E^{*} \rightarrow C^{*}(G)^{*}$ is a weakly compact operator, and we have $\langle T(f \circ \varphi), \omega(s)\rangle=f(\varphi(s))=\left\langle\Phi^{*} f, \omega(s)\right\rangle$ for all $f \in E^{*}, \quad s \in G$, so that $T(f \circ \varphi)=\Phi^{*} f$. Thus the set $\left\{T(f \circ \varphi) \mid f \in E^{*},\|f\| \leq 1\right\}$ is relatively weakly compact in $C^{*}(G)^{*}$. Furthermore, $\|\Phi\|=\left\|\Phi^{*}\right\|=\sup \left\{\|T(f \circ \varphi)\| \mid f \in E^{*},\|f\| \leq 1\right\}$. Suppose, conversely, that $f \circ \varphi \in B(G)$ for all $f \in E^{*}$, and that $\left\{T(f \circ \varphi) \mid f \in E^{*},\|f\| \leq 1\right\}$ is relatively weakly compact. Then we have a weakly compact operator $f \mapsto T(f \circ \varphi)$ from $E^{*}$ to $C^{*}(G)^{*}$. Its adjoint $S: C^{*}(G)^{* *} \rightarrow E^{* *}$ is weakly compact by Gantmacher's theorem, and continuous from $\sigma\left(C^{*}(G)^{* *}, C^{*}(G)^{*}\right)$ to $\sigma\left(E^{* *}, E^{* * *}\right)$ [6, p. 484]. If $s \in G$ and $f \in E^{*}$, then $\langle S(\omega(s)), f\rangle=\langle\omega(s), T(f \circ \varphi)\rangle=\langle\varphi(s), f\rangle$. 
Thus $S\left(\omega\left(M_{d d}(G)\right) \subset E\right.$. Since $M_{d d}(G)$ is weak* dense in $C^{*}(G)^{* *}$, and $E$, being a norm closed convex subset of $E^{* *}$, is $\sigma\left(E^{* *}, E^{* * *}\right)$-closed in $E^{* *}$, we have $S\left(C^{*}(G)^{* *}\right) \subset E$, so that $S$ may be regarded as a weakly compact operator from $C^{*}(G)^{* *}$ to $E$. Write now $\Phi=S \circ \varkappa$, where $x: C^{*}(G) \rightarrow C^{*}(G)^{* *}$ is the canonical embedding. Then $\Phi$ is a weakly compact operator. Both $\Phi^{* *}: C^{*}(G)^{* *} \rightarrow E$ and $S$ are continuous from $\sigma\left(C^{*}(G)^{* *}, C^{*}(G)^{*}\right)$ to $\sigma\left(E, E^{*}\right)$, and since they coincide on the weak* dense subspace $x\left(C^{*}(G)\right)$ of $C^{*}(G)^{* *}, \Phi^{* *}=S$. In particular, $\hat{\Phi}(s)=$ $S(\omega(s))=\varphi(s)$ for all $s \in G$, and the proof is complete.

In $[14$, p. 266] it is shown that if $\varphi: G \rightarrow E$ is bounded and weakly $\mu$-measurable for some $\mu \in M(G)$ (for example, weakly continuous), then there is a (clearly unique) element $\int \varphi d \mu \in E$ satisfying

$$
\left\langle\int \varphi d \mu, f\right\rangle=\int\langle\varphi(s), f\rangle d \mu(s) \text { for all } f \in E^{*} .
$$

Thus the symbol $\int \varphi d \mu$, which appears e.g. in the following lemma and theorem, is well defined. We mention in passing that in the situations which we encounter quite an elementary approach would suffice to define $\int \varphi d \mu$. One could consider a weak integral of $\varphi$ as an element of $E^{* *}$, and then conclude by weak compactness arguments that actually it belongs to $E$.

$\mathrm{L}$ e $\mathrm{m} \mathrm{m}$ a 3.4. The Fourier transform $\hat{\Phi}$ of any weakly compact operator $\Phi: C^{*}(G) \rightarrow E$ satisfies

$$
\int \hat{\Phi} d \mu=\Phi^{* *}(\omega(\mu))
$$

for all $\mu \in M(G)$.

Proof. For an arbitrary $\mu \in M(G)$ there is a net $\left(\mu_{\imath}\right)_{i \in I}$ in $M_{d d}(G)$ such that

$$
\lim _{i} \omega\left(\mu_{i}\right)=\omega(\mu)
$$

with respect to $\sigma\left(C^{*}(G)^{* *}, C^{*}(G)^{*}\right)$. As (1) holds for all $\mu_{i}$ in place of $\mu$ (in fact, if

$$
\left.v=\sum_{j=1}^{n} z_{j} \delta_{s_{j}} \in M_{d d}(G) \text {, then } \int \hat{\Phi} d v=\sum_{j=1}^{n} z_{j} \hat{\Phi}\left(s_{j}\right)=\Phi^{* *}(\omega(v))\right),
$$

the following simple passage to the limit proves it for $\mu$. Since $\Phi^{* *}: C^{*}(G)^{* *} \rightarrow E$ is continuous from $\sigma\left(C^{*}(G)^{* *}, C^{*}(G)^{*}\right)$ to $\sigma\left(E, E^{*}\right)$, and $f \circ \hat{\Phi} \in B(G)$ for all $f \in E^{*}$ (Theorem 3.3.) we get 


$$
\begin{aligned}
\left\langle\int \hat{\Phi} d \mu, f\right\rangle & =\int(f \circ \hat{\Phi}) d \mu=\langle\omega(\mu), T(f \circ \hat{\Phi})\rangle \\
& =\lim _{i}\left\langle\omega\left(\mu_{i}\right), T(f \circ \hat{\Phi})\right\rangle=\lim _{i}\left\langle\int \hat{\Phi} d \mu_{i}, f\right\rangle \\
& =\lim _{i}\left\langle\Phi^{* *}\left(\omega\left(\mu_{i}\right)\right), f\right\rangle=\left\langle\Phi^{* *}(\omega(\mu)), f\right\rangle \text {, i.e. (1) holds. }
\end{aligned}
$$

Th e o r e m 3.5. For a weakly continuous bounded function $\varphi: G \rightarrow E$ the following four conditions are equivalent:

(i) $\varphi$ is the Fourier transform of some weakly compact operator $\Phi: C^{*}(G) \rightarrow E$;

(ii) $\left\{\int \varphi d \mu \mid \mu \in M(G),\|\mu\|^{\prime} \leq 1\right\}$ is relatively weakly compact in $E$;

(iii) $\left\{\int \varphi d \mu \mid \mu \in M_{d d}(G),\|\mu\|^{\prime} \leq 1\right\}$ is relatively weakly compact in $E$;

(iv) $\left\{\int \varphi d \mu \mid \mu \in L^{1}(G),\|\mu\|^{\prime} \leq 1\right\}$ is relatively weakly compact in $E$.

If these equivalent conditions are satisfied, then $\|\Phi\|$ is equal to the supremum of the norms of the elements in any one of the sets appearing in (ii), (iii) and (iv).

Proof. Assume first (i). We may regard $\Phi^{* *}$ as a weakly compact operator from $C^{*}(G)^{* *}$ to $E$ (see [6, pp. 482-485]). Combined with Lemma 3.4 this shows that (ii) holds. Clearly, (ii) implies both (iii) and (iv). Assume now (iii). We intend to prove (i) by applying Theorem 3.3. From Lemma 2.1 it follows that $f \circ \varphi \in B(G)$ for all $f \in E^{*}$, because by assumption $f \circ \varphi$ is continuous, and the set

$$
\left\{\int(f \circ \varphi) d \mu \mid \mu \in M_{d d}(G),\|\mu\|^{\prime} \leq 1\right\}
$$

is bounded. To prove that $\left\{T(f \circ \varphi) \mid f \in E^{*},\|f\| \leq 1\right\}$ is relatively weakly compact in $C^{*}(G)^{*}$ we proceed as follows. Let $\psi: E^{*} \rightarrow C^{*}(G)^{*}$ be defined by $\psi(f)=T(f \circ \varphi)$. Let $F$ denote the norm closure of $\omega\left(M_{d d}(G)\right)$ in $C^{*}(G) * *$. Each $g \in C^{*}(G)^{*}$ determines a functional $\Theta g \in F^{*}$ by $\langle\Theta g, u\rangle=\langle u, g\rangle, u \in F$. Since the unit ball of the weak* dense sub- $C^{*}$-algebra $F$ of $C^{*}(G)^{* *}$ is by Kaplansky's density theorem weak* dense in the unit ball of $C^{*}(G)^{* *}$, the linear operator $\Theta: C^{*}(G)^{*} \rightarrow F^{*}$ is an isometry. Thus $\Theta\left(C^{*}(G)^{*}\right)$ is norm closed, hence $\sigma\left(F^{*}, F^{* *}\right)$-closed in $F^{*}$, and since $\sigma\left(F^{*}, F^{* *}\right)$ restricted to $\Theta\left(C^{*}(G)^{*}\right)$ corresponds (by the Hahn-Banach theorem) via $\Theta$ to $\sigma\left(C^{*}(G)^{*}, C^{*}(G)^{* *}\right)$, any set in $C^{*}(G)^{*}$ is relatively weakly compact if its image under $\Theta$ is so. Let now $S: \omega\left(M_{d d}(G)\right) \rightarrow E$ be defined by $S(\omega(\mu))=\int \varphi d \mu, \mu \in M_{d d}(G)$ (as $\omega: M(G) \rightarrow C^{*}(G)^{* *}$ is injective, this makes sense). By assumption, $S$ 
can be extended to a weakly compact operator from $F$ to $E$, also denoted by $S$. For all $f \in E^{*}$ we have

$$
\begin{aligned}
\left\langle S^{*} f, \omega(\mu)\right\rangle & =\langle f, S(\omega(\mu))\rangle=\left\langle f, \int \varphi d \mu\right\rangle=\int(f \circ \varphi) d \mu \\
& =\langle\omega(\mu), T(f \circ \varphi)\rangle=\langle\omega(\mu), \psi(f)\rangle \text { if } \mu \in M_{d d}(G),
\end{aligned}
$$

so that $S^{*} f=\Theta(\psi(f))$. Since $S^{*}$ is by Gantmacher's theorem a weakly compact operator, the set $\left\{\Theta(\psi(f)) \mid f \in E^{*},\|f\| \leq 1\right\}$, and hence the set $\left\{\psi(f) \mid f \in E^{*},\|f\| \leq 1\right\}=\left\{T(f \circ \varphi) \mid f \in E^{*},\|f\| \leq 1\right\}, \quad$ is relatively weakly compact. By Theorem 3.3, (i) holds, i.e. $\varphi=\hat{\Phi}$ for some weakly compact operator $\Phi: C^{*}(G) \rightarrow E$. Furthermore, applying Theorem 3.3 and the fact that $\left\|S^{*} f\right\|=\|\Theta(\psi(f))\|=\|T(f \circ \varphi)\|$ for all $f \in E^{*}$, we get

$$
\begin{gathered}
\|\Phi\|=\sup \left\{\|T(f \circ \varphi)\| \mid f \in E^{*},\|f\| \leq 1\right\} \\
=\left\|S^{*}\right\|=\|S\|=\sup \left\{\left\|\int \varphi d \mu\right\| \mid \mu \in M_{d d}(G),\|\mu\|^{\prime} \leq 1\right\} .
\end{gathered}
$$

Assume next (iv). Then the mapping $\mu \mapsto \int \varphi d \mu, \mu \in L^{1}(G)$, can be extended to a weakly compact operator $\Phi: C^{*}(G) \rightarrow E$. We claim that $\hat{\Phi}=\varphi$. For all $\mu \in L^{1}(G) \quad\left(\subset C^{*}(G)\right)$ we have $\Phi(\mu)=\Phi^{* *}(\omega(\mu))$, because $\omega(\mu)=\varkappa(\mu)$, where $\varkappa: C^{*}(G) \rightarrow C^{*}(G)^{* *}$ is the canonical embedding. Thus Lemma 3.4 shows that

$$
\begin{aligned}
\int(f \circ \varphi) d \mu & =\langle\Phi(\mu), f\rangle \\
& =\int(f \circ \hat{\Phi}) d \mu \text { for all } \mu \in L^{1}(G), f \in E^{*}
\end{aligned}
$$

As $f \circ \varphi$ and $f \circ \hat{\Phi}$ are continuous, the equation $\langle f, \varphi(s)\rangle=\langle f, \hat{\Phi}(s)\rangle$ is valid for all $s \in G, f \in E^{*}$, i.e. $\hat{\Phi}=\varphi$, so that (i) holds. Using again Lemma 3.4 we get

$$
\begin{aligned}
\|\Phi\| & =\sup \left\{\left\|\int \varphi d \mu\right\| \mid \mu \in L^{1}(G),\|\mu\|^{\prime} \leq 1\right\} \\
& \leq \sup \left\{\left\|\int \varphi d \mu\right\| \mid \mu \in M(G),\|\mu\|^{\prime} \leq 1\right\} \leq\left\|\Phi^{* *}\right\|=\|\Phi\|,
\end{aligned}
$$

which completes the proof.

Corollary 3.6. If $\varphi: G \rightarrow E$ is the Fourier transform of some weakly compact operator from $C^{*}(G)$ to $E$, the same is true of the function $s \mapsto \varphi\left(s^{-1}\right), \quad s \in G$. 
Proof. Since $s \mapsto \varphi\left(s^{-1}\right)$ is weakly (even strongly) continuous, it is in view of the above theorem sufficient to show that

$$
\|\mu\|^{\prime}=\left\|\sum_{j=1}^{n} z_{j} \delta_{s_{j}^{-1}}\right\|^{\prime} \text { for all } \mu=\sum_{j=1}^{n} z_{j} \delta_{s_{j}} \in M_{d d}(G) .
$$

Since $\bar{f} \in B(G)$ for any $f \in B(G)$, and $\|T \bar{f}\|=\|T f\|[8, \mathrm{p} .197]$, we get

$$
\begin{aligned}
\|\mu\|^{\prime} & =\|\omega(\mu)\|=\left\|\omega(\mu)^{*}\right\| \\
& =\sup \left\{\left|\sum_{j=1}^{n} \bar{z}_{j} f\left(s_{j}^{-1}\right)\right| \mid f \in B(G),\|T f\| \leq 1\right\} \\
& =\sup \left\{\left|\sum_{j=1}^{n} z_{j} \overline{f\left(s_{j}^{-1}\right)}\right| \mid f \in B(G),\|T f\| \leq 1\right\} \\
& =\sup \left\{\left|\sum_{j=1}^{n} z_{j} f\left(s_{j}^{-1}\right)\right| \mid f \in B(G),\|T f\| \leq 1\right\}=\left\|\sum_{j=1}^{n} z_{j} \delta_{s_{j}-1}\right\|^{\prime} .
\end{aligned}
$$

\section{Bounded bilinear forms on $C^{*}\left(G_{1}\right) \times C^{*}\left(G_{2}\right)$, and their Fourier transforms}

The following lemma is well known (it is mentioned without proof in $[11$, p. 5]). We give a proof for the sake of completeness.

$\mathrm{L} \mathrm{e} \mathrm{m} \mathrm{m}$ a 4.1. Let $E$ and $F$ be Banach spaces and $B: E \times F \rightarrow \mathbf{C}$ a bounded bilinear form. Let the operators $B_{l}: E \rightarrow F^{*}$ and $B_{r}: F \rightarrow E^{*}$ be defined by $\left\langle B_{l} x, y\right\rangle=\langle x, B, y\rangle=B(x, y)$ for $x \in E, \quad y \in F$. The following five conditions are equivalent:

(i) $\left\langle B_{l}^{* *} u, v\right\rangle=\left\langle u, B_{r}^{* *} v\right\rangle$ for all $u \in E^{* *}, v \in F^{* *}$;

(ii) (resp. (iii)) $B_{l}$ (resp. $B_{r}$ ) is a weakly compact operator;

(iv) (resp. (v)) the bilinear form $(u, v) \mapsto\left\langle B_{l}^{* *} u, v\right\rangle \quad(\operatorname{resp} . \quad(u, v) \mapsto$ $\left\langle u, B_{r}^{* *} v\right\rangle$ ) on $E^{* *} \times F^{* *}$ is separately weak* continuous.

If these equivalent conditions are satisfied, then $\tilde{B}: E^{* *} \times F^{* *} \rightarrow \mathbf{C}$ defined by $\tilde{B}(u, v)=\left\langle B_{l}^{* *} u, v\right\rangle=\left\langle u, B_{r}^{* *} v\right\rangle, \quad u \in E^{* *}, \quad v \in F^{* *}$, is the only separately weak* continuous bilinear form on $E^{* *} \times F^{* *}$ satisfying $\tilde{B}(\tilde{x}, \tilde{y})=B(x, y)$ for all $x \in E, \quad y \in F$, and their canonical images $\tilde{x} \in E^{* *}, \tilde{y} \in F^{* *}$. Furthermore, $\|\tilde{B}\|=\|B\|$.

Proof. We shall establish the following chain of implications: (i) $\Rightarrow$ (ii) $\Rightarrow$ (iv) $\Rightarrow($ ii $) \Rightarrow$ (i). The implications (i) $\Rightarrow($ iii $) \Rightarrow(v) \Rightarrow($ (iii $) \Rightarrow($ i) can be proved similarly. Assume first (i). Then $\left\langle B_{l}^{* *} u, v\right\rangle=\left\langle u, B_{r}^{* *} v\right\rangle=$ $\left\langle B_{r}^{*} u, v\right\rangle$ for all $u \in E^{* *}, v \in F^{* *}$, so that $B_{l}^{* *}\left(E^{* *}\right)=B_{r}^{*}\left(E^{* *}\right) \subset F^{*}$. This yields (ii) by Theorem 2 in [6, p. 482]. Next, assume (ii). Again we apply Theorem 2 in $\left[6\right.$, p. 482], and conclude that $B_{l}^{* *}\left(E^{* *}\right) \subset F^{*}$. Therefore the functional $v \mapsto\left\langle B_{l}^{* *} u, v\right\rangle$ on $F^{* *}$ is $\sigma\left(F^{* *}, F^{*}\right)$-continuous 
for each $u \in E^{* *}$. The $\sigma\left(E^{* *}, E^{*}\right)$-continuity of $u \mapsto\left\langle B_{l}^{* *} u, v\right\rangle$ on $E^{* *}$ for each $v \in F^{* *}$ is immediate. Thus (iv) holds. Assume now (iv). Since the functional $v \mapsto\left\langle u, B_{l}^{*} v\right\rangle=\left\langle B_{l}^{* *} u, v\right\rangle$ on $F^{* *}$ is $\sigma\left(F^{* *}, F^{*}\right)$ continuous for every $u \in E^{* *}, B_{l}^{*}: F^{* *} \rightarrow E^{*}$ is continuous from $\sigma\left(F^{* *}, F^{*}\right)$ to $\sigma\left(E^{*}, E^{* *}\right)$. By Lemma 7 in $[6$, p. 484], (ii) holds. Assume (ii) once more. This time we prove (i). Since $B_{r}=B_{l}^{*} \mid F$ is a weakly compact operator [6, pp. 484-485], (v) is obtained in the same way as (iv) was deduced from (ii) above. Since (the canonical image of) $E$ (resp. $F$ ) is weak* dense in $E^{* *}$ (resp. $\left.F^{* *}\right)$, we get (i) by applying (iv) and (v) together with the fact that $\left\langle B_{l}^{* *} \tilde{x}, \tilde{y}\right\rangle=B(x, y)=\left\langle\tilde{x}, B_{r}^{* *} \tilde{y}\right\rangle$ for all $x \in E, y \in F$. More generally, it is clear that there can be at most one separately weak* continuous bilinear form on $E^{* *} \times F^{* *}$ extending $B$. Finally, $\|\tilde{B}\|=\left\|B_{l}^{* *}\right\|=\left\|B_{l}\right\|=\|B\|$.

$\mathrm{D}$ efinition 4.2. If the five equivalent conditions in Lemma 4.1 are satisfied, then the bilinear form $\tilde{B}: E^{* *} \times F^{* *} \rightarrow \mathbf{C}$ defined there is called the canonical extension of $B$.

Remark 4.3. C. A. Akemann [1, p. 293] has proved that an arbitrary bounded linear map from a $C^{*}$-algebra to the predual of a von Neumann algebra is a weakly compact operator. In particular, if $A_{1}$ and $A_{2}$ are $C^{*}$-algebras, then any bounded linear operator from $A_{1}$ to $A_{2}^{*}$ is weakly compact, because $A_{2}^{* *}$ is a von Neumann algebra. Therefore the canonical extension $\tilde{B}$ of any bounded bilinear form $B: A_{1} \times A_{2} \rightarrow \mathbf{C}$ exists.

Throughout this section, $G_{1}$ and $G_{2}$ will be arbitrary locally compact groups. We are now able to define the Fourier transform of a bounded bilinear form on $C^{*}\left(G_{1}\right) \times C^{*}\left(G_{2}\right)$. The motivation for our use of this term will become clear in the next section, where the case of Abelian groups is discussed.

Definition 4.4. Let $B: C^{*}\left(G_{1}\right) \times C^{*}\left(G_{2}\right) \rightarrow \mathbf{C}$ be a bounded bilinear form. If $\tilde{B}: C^{*}\left(G_{1}\right)^{* *} \times C^{*}\left(G_{2}\right)^{* *} \rightarrow \mathbf{C}$ is its canonical extension, the function $\hat{B}: G_{1} \times G_{2} \rightarrow \mathbf{C}$ defined by $\hat{B}(s, t)=\tilde{B}\left(\omega_{1}(s), \omega_{2}(t)\right)$, $s \in G_{1}, t \in G_{2}$, is called the Fourier transform of $B$.

It is obvious in view of the continuity properties of $\omega_{j}$ and $\tilde{B}$ that $\hat{B}$ is separately continuous. The next theorem, which is a counterpart of Theorem 3.2, says that $B$ is even jointly continuous.

$\mathrm{Th}$ e o r e m 4.5. Let $B: C^{*}\left(G_{1}\right) \times C^{*}\left(G_{2}\right) \rightarrow \mathrm{C}$ be a bounded bilinear form. Then $\hat{B}$ is continuous on $G_{1} \times G_{2}$ (equipped with the product topology), and $|\hat{B}(s, t)| \leq\|B\|$ for all $s \in G_{1}, \quad t \in G_{2}$. If $B^{\prime}: C^{*}\left(G_{1}\right) \times$ $C^{*}\left(G_{2}\right) \rightarrow \mathbf{C}$ is any bounded bilinear form whose Fourier transform equals $\hat{B}$, then $B^{\prime}=B$.

Proof. Let $B_{l}: C^{*}\left(G_{1}\right) \rightarrow C^{*}\left(G_{2}\right)^{*}$ be defined by $\left\langle B_{l} x, y\right\rangle=$ 
$B(x, y), x \in C^{*}\left(G_{1}\right), y \in C^{*}\left(G_{2}\right)$. Since $B_{l}$ is a weakly compact operator by Remark 4.3, it has a Fourier transform $\hat{B}_{l}: G_{1} \rightarrow C^{*}\left(G_{2}\right)^{*}$. By definition, $\hat{B}(s, t)=\left\langle B_{l}^{* *} \omega_{1}(s), \omega_{2}(t)\right\rangle=\left\langle\hat{B_{l}}(s), \omega_{2}(t)\right\rangle=T_{2}^{-1}\left(\hat{B_{l}}(s)\right)(t)$ for all $s \in G_{1}, t \in G_{2}$. Using Theorem 3.2 and the fact that $\left\|T_{2}^{-1}\right\| \leq 1$ (which follows from the inequality $\|\mu\|^{\prime} \leq\|\mu\|, \mu \in L^{1}\left(G_{2}\right)$ ), we see that $\hat{B}(s, \cdot) \in B\left(G_{2}\right)$ for all $s \in G_{1}$ and that the map $s \mapsto \hat{B}(s, \cdot)$ is continuous with respect to the supremum norm topology of $B\left(G_{2}\right)$. Since each function in $B\left(G_{2}\right)$ is continuous, it follows easily that $\hat{B}$ is continuous. We have $\mid \hat{B}(s, t) \leq\|B\|\left\|\omega_{1}(s)\right\|\left\|\omega_{2}(t)\right\|=\|B\|, \quad s \in G_{1}, \quad t \in G_{2}$. The uniqueness statement is a consequence of the fact that $\omega_{j}\left(M_{d d}\left(G_{j}\right)\right)$ is weak* dense in $C^{*}\left(G_{j}\right)^{* *}, \mathrm{j}=1,2$, and $\tilde{B}$ is separately weak* continuous.

We shall follow I. Glicksberg [9] in giving a sense to the expression

$$
\iint K(s, t) d \mu(s) d v(t)
$$

where $K$ is a bounded separately continuous complex function on $G_{1} \times G_{2}$, and $\mu \in M\left(G_{1}\right), \quad v \in M\left(G_{2}\right)$. In fact, the functions

$$
t \mapsto \int K(s, t) d \mu(s), \quad t \in G_{2}, \text { and } s \mapsto \int K(s, t) d v(t), \quad s \in G_{1},
$$

are bounded and continuous, and

$$
\int\left(\int K(s, t) d \mu(s)\right) d v(t)=\int\left(\int K(s, t) d v(t)\right) d \mu(s)
$$

(see [9, pp. 205-208]). The common value of the two sides of this equation will be denoted by

$$
\iint K(s, t) d \mu(s) d v(t)
$$

The integration of bounded separately continuous functions is not really needed until Theorem 4.7, but we introduced it here to stress the fact that the next lemma is independent of the joint continuity of $\hat{B}$.

$\mathrm{L}$ e $\mathrm{mm}$ a 4.6. The Fourier transform $\hat{B}$ and canonical extension $\tilde{B}$ of any bounded bilinear form $B: C^{*}\left(G_{1}\right) \times C^{*}\left(G_{2}\right) \rightarrow \mathbf{C}$ satisfy $\iint \hat{B}(s, t) d \mu(s) d v(t)=\tilde{B}\left(\omega_{1}(\mu), \omega_{2}(v)\right)$ for all $\quad \mu \in M\left(G_{1}\right), \quad v \in M\left(G_{2}\right)$.

Proof. The operators $B_{l}: C^{*}\left(G_{1}\right) \rightarrow C^{*}\left(G_{2}\right)^{*}$ and $B_{r}: C^{*}\left(G_{2}\right) \rightarrow C^{*}\left(G_{1}\right)^{*}$ introduced in Lemma 4.1 are weakly compact (Remark 4.3). Thus $B_{r}^{* *}\left(\omega_{2}(t)\right) \in C^{*}\left(G_{1}\right)^{*}$ for all $t \in G_{2} \quad[6$, p. 482], and similarly

$$
B_{l}^{* *}\left(\omega_{1}(\mu)\right) \in C^{*}\left(G_{2}\right)^{*} \text {. }
$$


Since

$$
\hat{B}(s, t)=\left\langle\omega_{1}(s), B_{r}^{* *} \omega_{2}(t)\right\rangle, \quad s \in G_{1}, t \in G_{2},
$$

we obtain

$$
\left\langle B_{l}^{* *} \omega_{1}(\mu), \omega_{2}(t)\right\rangle=\left\langle\omega_{1}(\mu), B_{r}^{* *} \omega_{2}(t)\right\rangle=\int \hat{B}(s, t) d \mu(s)
$$

by Lemmas 4.1 and 2.2 , and so

$$
\hat{B}\left(\omega_{1}(\mu), \omega_{2}(v)\right)=\left\langle B_{l}^{* *} \omega_{1}(\mu), \omega_{2}(v)\right\rangle=\int\left(\int \hat{B}(s, t) d \mu(s)\right) d v(t) .
$$

We are now ready to characterize the Fourier transforms of bounded bilinear forms on $C^{*}\left(G_{1}\right) \times C^{*}\left(G_{2}\right)$.

Th e or e m 4.7. Let $K: G_{1} \times G_{2} \rightarrow \mathbf{C}$ be a bounded separately continuous function, and consider the inequality

$$
\left|\iint K(s, t) d \mu(s) d v(t)\right| \leq C\|\mu\|^{\prime}\|v\|^{\prime},
$$

where $\mu \in M\left(G_{1}\right), v \in M\left(G_{2}\right)$, and $C$ is a positive constant. The following six conditions are equivalent:

(i) $K$ is the Fourier transform of some bounded bilinear form $B: C^{*}\left(G_{1}\right) \times$ $C^{*}\left(G_{2}\right) \rightarrow \mathbf{C}$ with $\|B\| \leq C ;$

(ii) for all $\mu \in M\left(G_{1}\right)$ and $v \in M\left(G_{2}\right)$, (1) holds;

(iii) for all $\mu \in M_{d d}\left(G_{1}\right)$ and $v \in M_{d d}\left(G_{2}\right)$, (1) holds;

(iv) for all $\mu \in M_{d d}\left(G_{1}\right)$ and $v \in L^{1}\left(G_{2}\right)$, (1) holds;

(v) for all $\mu \in L^{1}\left(G_{1}\right)$ and $v \in M_{d d}\left(G_{2}\right)$, (1) holds;

(vi) for all $\mu \in L^{1}\left(G_{1}\right)$ and $v \in L^{1}\left(G_{2}\right)$, (1) holds.

Proof. It follows at once from Lemma 4.6 that (i) implies (ii). Clearly, (ii) implies all the statements from (iii) to (vi). Assume now (iii). For all $s \in G_{1}, K(s, \cdot)$ is a continuous function on $G_{2}$ and satisfies

$$
\left|\int K(s, t) d v(t)\right| \leq C\left\|\delta_{s}\right\|^{\prime}\|v\|^{\prime}=C\|v\|^{\prime}
$$

if $v \in M_{d d}\left(G_{2}\right)$, and so $K(s, \cdot) \in B\left(G_{2}\right)$ (Lemma 2.1). Define $\varphi: G_{1} \rightarrow$ $C^{*}\left(G_{2}\right) *$ by $\varphi(s)=T_{2}(K(s, \cdot))$. Then

$$
\begin{gathered}
\left\|\int \varphi d \mu\right\|=\sup \left\{\left|\int K(s, t) d \mu(s) d v(t)\right| \mid v \in M_{d d}\left(G_{2}\right),\right. \\
\left.\|v\|^{\prime} \leq 1\right\} \leq C\|\mu\|^{\prime}=C\left\|\omega_{1}(\mu)\right\|
\end{gathered}
$$

if $\mu \in M_{d d}\left(G_{1}\right)$ (see Lemma 2.1). As $\omega_{1}: M\left(G_{1}\right) \rightarrow C^{*}\left(G_{1}\right)^{* *}$ is injective there is a bounded linear map $F$ from the norm closure of $\omega_{1}\left(M_{d d}\left(G_{1}\right)\right)$ to $C^{*}\left(G_{2}\right)^{*}$ such that $F\left(\omega_{1}(\mu)\right)=\int \varphi(s) d \mu(s), \mu \in M_{d d}\left(G_{1}\right)$. Since the 
domain of $F$ is a $C^{*}$-algebra, it follows from the result of Akemann referred to in Remark 4.3 that $F$ is a weakly compact operator, so that $F\left(\omega_{1}\left(G_{1}\right)\right)$ is contained in some $\sigma\left(C^{*}\left(G_{2}\right)^{*}, C^{*}\left(G_{2}\right)^{* *}\right)$-compact subset of $C^{*}\left(G_{2}\right)^{*}$. On that set the topologies $\sigma\left(C^{*}\left(G_{2}\right)^{*}, C^{*}\left(G_{2}\right)^{* *}\right)$ and $\sigma\left(C^{*}\left(G_{2}\right)^{*}\right.$, $\left.\omega_{2}\left(M_{d d}\left(G_{2}\right)\right)\right)$ agree, because the latter is also Hausdorff, and coarser than the former. Thus $\varphi$ is weakly continuous, because it is by assumption (and Lemma 2.2) continuous with respect to $\sigma\left(C^{*}\left(G_{2}\right)^{*}, \omega_{2}\left(M_{d d}\left(G_{2}\right)\right)\right.$ ). Since $F$ is a weakly compact operator and $\|F\| \leq \mathrm{C}$, Theorem 3.5 yields a weakly compact operator $\Phi: C^{*}\left(G_{1}\right) \rightarrow C^{*}\left(G_{2}\right)^{*}$ such that $\|\Phi\| \leq C$ and $\varphi$ is the Fourier transform of $\Phi$. We now define a bounded bilinear form $B: C^{*}\left(G_{1}\right) \times C^{*}\left(G_{2}\right) \rightarrow \mathbf{C}$ by $B(x, y)=\langle\Phi x, y\rangle$. Then $\|B\|=$ $\|\Phi\| \leq C$, and for all $s \in G_{1}, t \in G_{2}$, we have

$$
\begin{aligned}
\hat{B}(s, t) & =\tilde{B}\left(\omega_{1}(s), \omega_{2}(t)\right) \\
& =\left\langle\Phi^{* *}\left(\omega_{1}(s)\right), \omega_{2}(t)\right\rangle=\left\langle\varphi(s), \omega_{2}(t)\right\rangle \\
& =\left\langle T_{2}\left(K(s, \cdot), \omega_{2}(t)\right\rangle=K(s, t),\right.
\end{aligned}
$$

i.e. (i) holds. A simple modification of the preceding argument yields a proof that (iv) implies (i). The only changes that need to be made concern the construction of $F$. Now we have

$$
\left|\int K(s, t) d v(t)\right| \leq C\left\|\delta_{s}\right\|^{\prime}\|v\|^{\prime} \text { for all } v \in L^{1}\left(G_{2}\right),
$$

so that $K(s, \cdot) \in B\left(G_{2}\right)$, and $\varphi: G_{1} \rightarrow C^{*}\left(G_{2}\right)^{*}$ can be defined by $\varphi(s)=$ $T_{2}(K(s, \cdot))$ as before. The proof that $\left\|\int \phi d \mu\right\| \leq C\|\mu\|^{\prime}, \mu \in M_{d d}\left(G_{1}\right)$ involves this time a supremum over the measures $v \in L^{1}\left(G_{2}\right)$ with $\|v\|^{\prime} \leq 1$. When the roles of $G_{1}$ and $G_{2}$ are exchanged, the same proof shows that $(\mathrm{v})$ implies (i). Finally, assume (vi). Then we get at once a bounded bilinear form $B: C^{*}\left(G_{1}\right) \times C^{*}\left(G_{2}\right) \rightarrow \mathbf{C}$ such that $\|B\| \leq C$ and

$$
\begin{gathered}
\tilde{B}\left(\omega_{1}(\mu), \omega_{2}(v)\right)=B(\mu, v)=\iint K(s, t) d \mu(s) d v(t) \text { for } \\
\mu \in L^{1}\left(G_{1}\right) \subset C^{*}\left(G_{1}\right), v \in L^{1}\left(G_{2}\right) \subset C^{*}\left(G_{2}\right)
\end{gathered}
$$

(recall that e.g. $\omega_{1}(\mu)$ is simply the canonical image of $\mu$ in $C^{*}\left(G_{1}\right)^{* *}$ ) . By Lemma 4.6,

$$
\begin{gathered}
\iint \hat{B}(s, t) d \mu(s) d v(t)=\iint K(s, t) d \mu(s) d v(t) \text { for all } \\
\mu \in L^{1}\left(G_{1}\right), \quad v \in L^{1}\left(G_{2}\right) .
\end{gathered}
$$

Since the functions 


$$
t \mapsto \iint \hat{B}(s, t) d \mu(s) \text { and } t \mapsto \iint K(s, t) d \mu(s)
$$

are continuous on $G_{2}$ (see the discussion preceding Lemma 4.6), this shows that

$$
\int \hat{B}(s, t) d \mu(s)=\int K(s, t) d \mu(s) \text { for all } \mu \in L^{1}\left(G_{1}\right), t \in G_{2} .
$$

In view of the continuity of the functions $\hat{B}(\cdot, t)$ and $K(\cdot, t)$ we have therefore $\hat{B}(s, t)=K(s, t)$ for all $s \in G_{1}, t \in G_{2}$. Thus (i) holds, and the theorem is proved.

Corollary 4.8. If $K: G_{1} \times G_{2} \rightarrow \mathbf{C}$ is the Fourier transform of some bounded bilinear form on $C^{*}\left(G_{1}\right) \times C^{*}\left(G_{2}\right)$, the same is true of each one of the functions $(s, t) \mapsto K\left(s^{-1}, t\right), \quad(s, t) \mapsto K\left(s, t^{-1}\right)$ and $(s, t) \mapsto$ $K\left(s^{-1}, t^{-1}\right)$ on $G_{1} \times G_{2}$.

Proof. Since $K$ is bounded and continuous (only separate continuity is needed), the corollary follows from the above theorem and the fact that e.g.

$$
\left\|\sum_{j=1}^{n} z_{j} \delta_{s_{j}}\right\|^{\prime}=\left\|\sum_{j=1}^{n} z_{j} \delta_{s_{j}-1}\right\|^{\prime} \text { for all } \sum_{j=1}^{n} z_{j} \delta_{s_{j}} \in M_{d d}\left(G_{1}\right)
$$

(see the proof of Corollary 3.6).

In the rest of this section we discuss positive-definite bilinear forms (defined below). Such bilinear forms arise naturally in the study of stochastic processes (Section 6).

Definition 4.9. Let $A$ be a $C^{*}$-algebra and $B: A \times A \rightarrow \mathbf{C}$ a bilinear form. If $B\left(x, x^{*}\right) \geq 0$ for all $x \in A$, then $B$ is said to be positive-definite.

Th e o r e m 4.10. Let $M$ be a von Neumann algebra with predual $M_{*}$, and let $D$ be a $\sigma\left(M, M_{*}\right)$-dense *-subalgebra of $M$. For a separately $\sigma\left(M, M_{*}\right)$-continuous bilinear form $B: M \times M \rightarrow \mathbf{C}$ the following three conditions are equivalent:

(i) $B\left(x, x^{*}\right) \geq 0$ for all $x \in D$;

(ii) there is a Hilbert space $H$ and a bounded linear map $\Phi: D \rightarrow H$ such that $B(x, y)=\left(\Phi x \mid \Phi y^{*}\right)$ for all $x, y \in D$;

(iii) $B$ is positive-definite.

Proof. Assume first (i) and define $h(x, y)=B\left(x, y^{*}\right)$ for $x, y \in D$. Then $h$ is linear in the first argument and conjugate-linear in the second, and satisfies the polarization identity $4 h(x, y)=h(x+y, x+y)-$ $h(x-y, x-y)+i h(x+i y, x+i y)-i h(x-i y, x-i y)$, so that by our assumption $h(x, y)=\overline{h(y, x)}$ for all $x, y \in D$. Thus $h$ is a positive Hermitian form. A standard construction now yields the Hilbert space $H$ required for (ii). In fact, if $N=\{x \in D \mid h(x, x)=0\}$, the 
Cauchy-Schwarz inequality shows that $N$ is a linear subspace of $D$, and $H$ is then defined as the completion of the pre-Hilbert space $D / N$ equipped with the (in view of the Cauchy-Schwarz inequality well-defined) scalar product $(p x \mid p y)=h(x, y), x, y \in D$, where $p: D \rightarrow D / N$ is the quotient map. It is immediately seen that (ii) holds with $\Phi=p$ (the boundedness of $\Phi$ is a consequence of the well-known fact that $B$ is by virtue of the uniform boundedness principle a bounded bilinear form on $M \times M)$. Assume next (ii). We may suppose that $\Phi(D)$ is dense in $H$, and thus identify $\Phi(D)$ with a dense subspace $E$ of $H^{*}$ by defining $\langle\Phi x, \xi\rangle=(\xi \mid \Phi x), \quad x \in D, \quad \xi \in H$. Then $\Phi$ is by the separate $\sigma\left(M, M_{*}\right)$-continuity of $B$ continuous from $\sigma\left(D, M_{*}\right)$ to $\sigma(H, E)$. We shall consider $H$ embedded canonically into the algebraic dual $F$ of $E$. Since $F$ is known to be $\sigma(F, E)$-complete, $\Phi$ can be extended to a linear map $\tilde{\Phi}: M \rightarrow F$, which is continuous from $\sigma\left(M, M_{*}\right)$ to $\sigma(F, E)$. As every bounded set in a Hilbert space is relatively weakly compact, $\Phi$ maps the unit ball $D_{1}$ of $D$ into a $\sigma\left(H, H^{*}\right)$-compact subset $K$ of $H$. Since $K$ is also compact with respect to the coarser Hausdorff topology $\sigma(H, E)$, and $D_{1}$ is by Kaplansky's density theorem $\sigma\left(M, M_{*}\right)$-dense in the unit ball $M_{1}$ of $M, \tilde{\Phi}\left(M_{1}\right)$ is by continuity contained in (the canonical image of) $K$, and so $\tilde{\Phi}$ can be regarded as a bounded operator from $M$ into $H$. We now show that

$$
\left(\tilde{\Phi} x \mid \tilde{\Phi} y^{*}\right)=B(x, y) \text { for all } x, y \in M .
$$

Clearly we may assume that $\|x\| \leq 1,\|y\| \leq 1$. Since $\tilde{\Phi}$ is continuous from $\sigma\left(M, M_{*}\right)$ to $\sigma(H, E)$, and $\sigma(H, E)$ agrees with $\sigma\left(H, H^{*}\right)$ on bounded subsets of $H$ (recall that $E$ is norm dense in $H^{*}$ ), the restriction $\tilde{\Phi} \mid M_{1}$ is continuous from the relative $\sigma\left(M, M_{*}\right)$-topology to $\sigma\left(H, H^{*}\right)$. Since the involution in $M$ is $\sigma\left(M, M_{*}\right)$-continuous, the map $(u, v) \mapsto\left(\tilde{\Phi} u \mid \tilde{\Phi} v^{*}\right)$ is therefore separately $\sigma\left(M, M_{*}\right)$-continuous when restricted to $M_{1} \times M_{1}$. That map agrees with $B$ on $D_{1} \times D_{1}$. Using again the fact that $D_{1}$ is $\sigma\left(M, M_{*}\right)$-dense in $M_{1}$, we obtain the formula $\left(\tilde{\Phi} x \mid \tilde{\Phi} y^{*}\right)=B(x, y)$ for all $x$ and $y$ in $M_{1}$ (and in $M$ ). In particular, $B\left(x, x^{*}\right) \geq 0$ if $x \in M$, and so (iii) holds. As (iii) trivially implies (i), the theorem is proved.

Let us recall the following well-known definition.

Definition 4.11. For any set $X$, a function $K: X \times X \rightarrow \mathbf{C}$ is called a positive-definite kernel if

$$
\sum_{s \in X} \sum_{t \in X} a(s) \overline{a(t)} K(s, t) \geq 0
$$

for every finitely supported function $a: X \rightarrow \mathbf{C}$. 
Th e or e m 4.12. Let $A$ be a $C^{*}$-algebra. For a bounded bilinear form $B: A \times A \rightarrow \mathbf{C}$ the following three conditions are equivalent:

(i) $B$ is positive-definite;

(ii) there is a Hilbert space $H$ and a bounded linear map $\Phi: A \rightarrow H$ such that $B(x, y)=\left(\Phi x \mid \Phi y^{*}\right)$ for all $x, y \in A$;

(iii) the canonical extension $\tilde{B}$ of $B$ is positive-definite.

In case $A=C^{*}(G)$ for a locally compact group $G$, these equivalent conditions are satisfied if and only if for the Fourier transform $\hat{B}$ of $B$ the function $(s, t) \mapsto \hat{B}\left(s, t^{-1}\right)$ on $G \times G$ is a positive-definite kernel.

Proof. The equivalence of (i), (ii) and (iii) follows from Theorem 4.10, when $A$ is considered canonically embedded in $A^{* *}$. (Not nearly the full force of Theorem 4.10 is needed; since $\Phi: A \rightarrow H$ now has the obvious extension $\Phi^{* *}: A^{* *} \rightarrow H$, the use of Kaplansky's density theorem can be avoided.) Since

$$
\sum_{s \in G} \sum_{t \in G} a(s) \overline{a(t)} \hat{B}\left(s, t^{-1}\right)=\tilde{B}\left(\omega\left(\sum_{s \in G} a(s) \delta_{s}\right), \omega\left(\sum_{t \in G} a(t) \delta_{t}\right)^{*}\right)
$$

for every finitely supported function $a: G \rightarrow \mathbf{C}$, and $\omega\left(M_{d d}(G)\right)$ is a weak* dense *-subalgebra of $C^{*}(G)^{* *}$, the second assertion is also a consequence of Theorem 4.10.

\section{Fourier transforms of regular Borel vector measures and bounded bimeasures}

The results in Sections 3 and 4 involving a locally compact group $G$, or two such groups $G_{1}$ and $G_{2}$, take on a more palpable appearance if these groups happen to be commutative. The present section is devoted to a study of the commutative case. Throughout the rest of this section, $E$ is a Banach space, $G, G_{1}$ and $G_{2}$ are locally compact Abelian groups, and their dual groups are denoted by $\Gamma, \Gamma_{1}$ and $\Gamma_{2}$, respectively. We continue to use the multiplicative notation for the group operations. The value of a character $\gamma \in \Gamma$ at $s \in G$ is denoted by $(s, \gamma)$.

We begin with a lemma dealing with two natural ways of identifying $C^{*}(G)$ with $C_{0}(\Gamma)$. For each $\mu \in M(G)$, the functions $\hat{\mu}: \Gamma \rightarrow \mathbf{C}$ and $\check{\mu}: \Gamma \rightarrow \mathbf{C}$ are defined by

$$
\hat{\mu}(\gamma)=\int_{G} \overline{(s, \gamma)} d \mu(s) \text { and } \stackrel{\nu}{\mu}(\gamma)=\int_{G}(s, \gamma) d \mu(s) .
$$

Similarly, we write 


$$
\begin{gathered}
\hat{v}(s)=\int_{\Gamma} \overline{(s, \gamma)} d v(\gamma) \text { and } \\
\check{v}(s)=\int_{\Gamma}(s, \gamma) d v(\gamma) \text { for } v \in M(\Gamma), s \in G .
\end{gathered}
$$

$\mathrm{L}$ e $\mathrm{m} \mathrm{m}$ a 5.1 . There is a unique isometric *isomorphism $\alpha: C^{*}(G) \rightarrow$ $C_{0}(T) \quad\left(\operatorname{resp} . \quad \beta: C^{*}(G) \rightarrow C_{0}(\Gamma)\right)$ such that $\alpha(\mu)=\hat{\mu} \quad(\operatorname{resp} . \quad \beta(\mu)=\breve{\mu})$ for all $\mu \in L^{1}(G) \subset C^{*}(G)$. We have $\alpha^{*}(v)=T(\hat{v})$ and $\beta^{*}(v)=T(\grave{v})$ for all $v \in M(\Gamma)$. If $\sigma: C_{0}(\Gamma) \rightarrow C_{0}(\Gamma)$ is the isomorphism satisfying $\sigma(f)(\gamma)=$ $f\left(\gamma^{-1}\right)$ for $f \in C_{0}(\Gamma), \gamma \in \Gamma$, then $\beta=\sigma \circ \alpha$.

Proof. The existence and uniqueness of $\alpha$ and $\beta$ follow from the definition of $C^{*}(G)$ and the equalities $\|\mu\|^{\prime}=\|\mu\|^{\wedge}=\|\hat{\mu}\|_{\infty}=\|\check{\mu}\|_{\infty}$, $\mu \in L^{1}(G)$, combined with the fact that both $\mu \mapsto \hat{\mu}$ and $\mu \mapsto \tilde{\mu}$ are *-homomorphisms from $L_{1}(G)$ onto a dense *-subalgebra of $C_{0}(\Gamma)$. Clearly, $\beta=\sigma \circ \alpha$. The equations $\alpha^{*}(v)=T(\hat{v})$ and $\beta^{*}(v)=T(\grave{v})$ follow from Fubini's theorem. For example, if $v \in M(\Gamma)$ and $\mu \in L^{1}(G) \subset C^{*}(G)$, we get

$$
\begin{aligned}
\int_{G} T^{-1} \alpha^{*}(v)(s) d \mu(s) & =\left\langle\alpha^{*}(v), \mu\right\rangle=\langle\nu, \alpha(\mu)\rangle \\
& =\int_{\Gamma}\left(\int_{G} \overline{(s, \gamma)} d \mu(s)\right) d v(\gamma)=\int_{G} \hat{v}(s) d \mu(s),
\end{aligned}
$$

so that $T^{-1} \alpha^{*}(v)=\hat{v}$.

When $C_{0}(\Gamma)$ takes the role of $C^{*}(G)$ in accordance with the above lemma, the two types of Fourier transforms discussed in Sections 3 and 4 can be introduced by means of integration. Let us first consider the case of a weakly compact operator from $C^{*}(G)$ to $E$.

Let $\mathscr{B}$ be the Borel $\sigma$-algebra of $\Gamma$ (i.e. the $\sigma$-algebra generated by the open subsets of $\Gamma$ ). We call any countably additive mapping $m: \mathscr{B} \rightarrow E$ a Borel vector measure on $\Gamma$. The set of all $E$-valued regular (for the definition, see e.g. [14, p. 262]) Borel vector measures on $\Gamma$ will be denoted by $M(\Gamma, E)$. The set of all weakly compact operators from $C_{0}(\Gamma)$ to $E$ is known to be via integration in a bijective correspondence with $M(T, E)$ (see e.g. Lemma 2 in $[14$, p. 264]). One may consult [6] and [14] as to details and references on the theory of integration with respect to vector measures. For example, if $g$ is a bounded continuous complex function on $\Gamma$, and $m \in M(\Gamma, E)$, there is a unique element $\int g d m \in E$ which satisfies

$$
\left\langle\int g d m, f\right\rangle=\int g d(f \circ m) \text { for all } f \in E^{*} .
$$


Let $\alpha$ and $\beta$ be as in Lemma 5.1. For each $m \in M(T, E)$, we let $m_{\alpha}$ (resp. $m_{\beta}$ ) stand for the weakly compact operator from $C^{*}(G)$ to $E$ defined by $m_{\alpha}(x)=\int \alpha(x) d m$ (resp. $m_{\beta}(x)=\int \beta(x) d m$ ) for all $x \in C^{*}(G)$. The Fourier transforms $\hat{m}_{\alpha}$ and $\hat{m}_{\beta}$ of $m_{\alpha}$ and $m_{\beta}$ (in the sense of Section 3 ) have the following interpretations:

$\mathrm{L}$ e $\mathrm{m} \mathrm{m}$ a 5.2 . If $m \in M(\Gamma, E)$, then

$$
\hat{m}_{\alpha}(s)=\int \overline{(s, \gamma)} d m(\gamma) \text { and } \hat{m}_{\beta}(s)=\int(s, \gamma) d m(\gamma) \text { for all } s \in G \text {. }
$$

Proof. By Lemma 5.1,

$$
\begin{aligned}
\left\langle\int \overline{(s, \gamma)} d m(\gamma), f\right\rangle & =\int \overline{(s, \gamma)} d(f \circ m)(\gamma)=T^{-1} \alpha^{*}(f \circ m)(s) \\
& =\left\langle\alpha^{*}(f \circ m), \omega(s)\right\rangle \text { for all } s \in G, f \in E^{*} .
\end{aligned}
$$

An elementary calculation shows that $\left\langle\alpha^{*}(f \circ m), \omega(s)\right\rangle=\left\langle m_{\alpha}^{* *}(\omega(s)), f\right\rangle$, and so

$$
\hat{m}_{\alpha}(s)=\int \overline{(s, \gamma)} d m(\gamma)
$$

The proof of the second formula is similar.

If $\Phi: C^{*}(G) \rightarrow E$ is a weakly compact operator, there is obviously exactly one $m \in M(\Gamma, E)$ (resp. $n \in M(\Gamma, E)$ ) such that $\Phi=m_{\alpha}$ (resp. $\Phi=n_{\beta}$ ). This fact along with Lemma 5.2 enables us to deduce from Theorems 3.2 and 3.5, and Corollary 3.6 the following result, which is due to I. Kluvánek (see [14], Theorem 2, its Corollary 3, Theorem 3, and the last paragraph in its proof.)

Th e o r e m 5.3. For a function $\varphi: G \rightarrow E$ the following four conditions are equivalent:

(i) (resp. (ii)) there is $m \in M(\Gamma, E)$ such that

$$
\varphi(s)=\int \overline{(s, \gamma)} d m(\gamma)\left(\text { resp. } \varphi(s)=\int(s, \gamma) d m(\gamma)\right) \text { for all } s \in G ;
$$

(iii) (resp. (iv)) $\varphi$ is bounded and weakly continuous, and one (resp. every one) of the sets $\left\{\int \varphi d \mu \mid \mu \in M(G),\|\hat{\mu}\|_{\infty} \leq 1\right\}, \quad\left\{\int \varphi d \mu \mid \mu \in M_{d d}(G)\right.$, $\left.\|\hat{\mu}\|_{\infty} \leq 1\right\}$ and $\left\{\int \varphi d \mu \mid \mu \in L^{1}(G),\|\hat{\mu}\|_{\infty} \leq 1\right\}$ is relatively weakly compact in $E$.

If these equivalent conditions hold, then $\varphi$ is strongly continuous.

The latter part of this section deals with the interpretation of the Fourier transform of a bounded bilinear form on $C^{*}\left(G_{1}\right) \times C^{*}\left(G_{2}\right)$ in the commutative case. One is led to consider the problem of extending a bounded bilinear form originally defined on $C_{0}\left(\Gamma_{1}\right) \times C_{0}\left(\Gamma_{2}\right)$ to other pairs of functions (in particular, continuous characters) on $\Gamma_{1}$ and $\Gamma_{2}$. 
Definition 5.4. Let $X$ and $Y$ be locally compact Hausdorff spaces and $B: C_{0}(X) \times C_{0}(Y) \rightarrow \mathrm{C}$ a bounded bilinear form. Then $B$ is called a bounded bimeasure on $X \times Y$. Let $f: X \rightarrow \mathbf{C}$ and $g: Y \rightarrow \mathbf{C}$ be bounded Borel functions, and define $\tilde{f} \in M(X)^{*}$ by

$$
\langle\tilde{f}, \mu\rangle=\int f d \mu \text { for } \mu \in M(X),
$$

and similarly $\tilde{g} \in M(Y)^{*}$. We use the notation

$$
B(f, g)=\tilde{B}(\tilde{f}, \tilde{g})
$$

where $\tilde{B}: C_{0}(X)^{* *} \times C_{0}(Y)^{* *} \rightarrow \mathbf{C}$ is the canonical extension of $B$, and call $B(f, g)$ the integral of the pair $(f, g)$ with respect to the bimeasure $B$.

Remark 5.5. The existence of $\tilde{B}$ was noted in Remark 4.3 (in the present situation it suffices to use a special case of the result of Akemann referred to in Remark 4.3, namely, the fact due to A. Grothendieck [10] that any bounded linear operator from $C_{0}(X)$ to $M(Y)$ is weakly compact). Obviously (1) agrees with the original meaning of $B(f, g)$ if $f \in C_{0}(X)$, $g \in C_{0}(Y)$. Let us observe that (1) is for any bounded Borel functions $f$ and $g$ consistent with the notation of E. Thomas [23] (transferred to the case of complex scalars). First of all, it is clear that $B(f, \cdot)$ and $B(\cdot, g)$ in the sense of $[23, \mathrm{p} .145]$ exist, and that $g$ is $B(f, \cdot)$-integrable and $f$ $B(\cdot, g)$-integrable. Here e.g. $B(\cdot, g) \in M(X)$ is the bounded linear functional

$$
h \mapsto \int g d B(h, \cdot)
$$

on $C_{0}(X)$ (where $B(h, \cdot)$ is of course the measure $k \mapsto B(h, k)$, $\left.k \in C_{0}(Y)\right)$. If $B_{l}: C_{0}(X) \rightarrow M(Y)$ is the operator $h \mapsto B(h, \cdot)$, then

$$
\left\langle B_{l}^{* *} \tilde{f}, \tilde{g}\right\rangle=\left\langle\tilde{f}, B_{l}^{*} \tilde{g}\right\rangle=\int f d B(\cdot, g),
$$

because $B_{l}^{*} \tilde{g}=B(\cdot, g)$ by virtue of the fact that

$$
\left\langle h, B_{l}^{*} \tilde{g}\right\rangle=\left\langle B_{l} h, \tilde{g}\right\rangle=\int g d B(h, \cdot) \text { for all } h \in C_{0}(X) .
$$

By a similar argument,

$$
\left\langle\tilde{f}, B_{r}^{* *} \tilde{g}\right\rangle=\int g d B(f, \cdot),
$$

where $\quad B_{r}: C_{0}(Y) \rightarrow M(X) \quad$ is defined by $\left\langle h, B_{r} k\right\rangle=B(h, k)$, $h \in C_{0}(X), \quad k \in C_{0}(Y)$. Thus the equality $\tilde{B}(\tilde{f}, \tilde{g})=\left\langle B_{l}^{* *} \tilde{f}, \tilde{g}\right\rangle=$ 
$\left\langle f, B_{r}^{* *} \tilde{g}\right\rangle$, which is guaranteed by Lemma 4.1 and the weak compactness of e.g. $B_{l}$, shows that the pair $(f, g)$ is $B$-integrable in the sense of $[23$, p. 145], and (1) gives the same value to $B(f, g)$ as the definition in [23, p. 145]. The (complex analogue of the) proposition in [23, p. 145] could also be used to prove the $B$-integrability of the pair $(f, g)$.

The following lemma relates the integral of a pair of continuous characters on $\Gamma_{1}$ and $\Gamma_{2}$ with respect to a bounded bimeasure on $\Gamma_{1} \times \Gamma_{2}$ to the Fourier transforms of certain bounded bilinear forms on $C^{*}\left(G_{1}\right) \times C^{*}\left(G_{2}\right)$. For any locally compact Abelian group $G$ and $s \in G$, $\left(s,{ }^{\circ}\right)$ denotes the continuous character $\gamma \mapsto(s, \gamma)$ on the dual group of $G$.

$\mathrm{L}$ e $\mathrm{mm}$ a 5.6. Let $B$ be a bounded bimeasure on $\Gamma_{1} \times \Gamma_{2}$. Let us define four bounded bilinear forms on $C^{*}\left(G_{1}\right) \times C^{*}\left(G_{2}\right)$ by

$$
\begin{aligned}
& B_{\alpha, \alpha}(x, y)=B\left(\alpha_{1}(x), \alpha_{2}(y)\right), B_{\alpha, \beta}(x, y)=B\left(\alpha_{1}(x), \beta_{2}(y)\right), \\
& B_{\beta, \alpha}(x, y)=B\left(\beta_{1}(x), \alpha_{2}(y)\right), B_{\beta, \beta}(x, y)=B\left(\beta_{1}(x), \beta_{2}(y)\right),
\end{aligned}
$$

where $\alpha_{j}, \beta_{j}: C^{*}\left(G_{j}\right) \rightarrow C_{0}\left(\Gamma_{j}\right), j=1,2$, correspond to the isomorphisms $\alpha$ and $\beta$ in Lemma 5.1. Then the Fourier transforms of these bilinear forms satisfy

$$
\begin{aligned}
B((s, \cdot),(t, \cdot)) & =\hat{B}_{\alpha, \alpha}\left(s^{-1}, t^{-1}\right)=\hat{B}_{\alpha, \beta}\left(s^{-1}, t\right) \\
& =\hat{B}_{\beta, \alpha}\left(s, t^{-1}\right)=\hat{B}_{\beta, \beta}(s, t) \text { for all } s \in G_{1}, t \in G_{2} .
\end{aligned}
$$

Proof. We prove, for example, the equation

$$
B((s, \cdot),(t, \cdot))=\hat{B}_{\alpha, \beta}\left(s^{-1}, t\right) .
$$

For $s \in G_{1}$, let $\tilde{s} \in M\left(\Gamma_{1}\right)^{*}$ be defined by

$$
\langle\tilde{s}, v\rangle=\int(s, \gamma) d v(\gamma), \quad v \in M\left(\Gamma_{1}\right) .
$$

Then $\tilde{s}=\alpha_{1}^{* *}\left(\omega_{1}\left(s^{-1}\right)\right)$, because by Lemma 5.1,

$$
\begin{aligned}
\left\langle\alpha_{1}^{* *}\left(\omega_{1}\left(s^{-1}\right)\right), v\right\rangle & =\left\langle\omega_{1}\left(s^{-1}\right), \alpha_{1}^{*}(v)\right\rangle=\left\langle\omega_{1}\left(s^{-1}\right), T_{1} \hat{v}\right\rangle \\
& =\hat{v}\left(s^{-1}\right)=\int(s, \gamma) d v(\gamma) \text { for all } v \in M\left(\Gamma_{1}\right) .
\end{aligned}
$$

Similarly, $\beta_{2}^{* *}\left(\omega_{2}(t)\right)=\tilde{t}$, if $t \in G_{2}$. It is easily seen that $\tilde{B}_{\alpha, \beta}(u, v)=$ $\tilde{B}\left(\alpha_{1}^{* *} u, \beta_{2}^{* *} v\right)$ for all $u \in C^{*}\left(G_{1}\right)^{* *}, \quad v \in C^{*}\left(G_{2}\right)^{* *}$, so that

$$
B((s, \cdot),(t, \cdot))=\tilde{B}(\tilde{s}, \tilde{t})=\tilde{B}_{\alpha, \beta}\left(\omega_{1}\left(s^{-1}\right), \omega_{2}(t)\right)=\hat{B}_{\alpha, \beta}\left(s^{-1}, t\right) .
$$

The next result is the commutative version of Theorem 4.7 in con- 
junction with Theorem 4.5 and Corollary 4.8. Its proof is an obvious application of the preceding lemma.

Theorem 5.7. For a function $K: G_{1} \times G_{2} \rightarrow \mathbf{C}$ and constant $C>0$, the following four conditions are equivalent:

(i) (resp. (ii)) one (resp. every one) of the formulas

$$
\begin{aligned}
& K(s, t)=B_{1}((s, \cdot),(t, \cdot)), \quad K(s, t)=B_{2}\left((s, \cdot),\left(t^{-1}, \cdot\right)\right), \\
& K(s, t)=B_{3}\left(\left(s^{-1}, \cdot\right),(t, \cdot)\right), \quad K(s, t)=B_{4}\left(\left(s^{-1}, \cdot\right),\left(t^{-1}, \cdot\right)\right),
\end{aligned}
$$

$s \in G_{1}, t \in G_{2}$, is valid for some bounded bimeasure

$$
B_{j}: C_{0}\left(\Gamma_{1}\right) \times C_{0}\left(\Gamma_{2}\right) \rightarrow \mathbf{C} \text { with }\left\|B_{j}\right\| \leq C, \quad j=1, \ldots, 4 ;
$$

(iii) (resp. (iv)) $K$ is bounded and separately continuous, and one (resp. every one) of the conditions (ii) through (vi) in Theorem 4.7 is satisfied when

$$
\|\mu\|^{\prime}=\|\hat{\mu}\|_{\infty}, \quad\|v\|^{\prime}=\|\hat{v}\|_{\infty} .
$$

If these equivalent conditions hold, then $K$ is continuous with respect to the product topology of $G_{1} \times G_{2}$.

The last theorem in this section characterizes positive-definite bounded bimeasures. Before stating it, let us recall the well-known fact that if $X$ is any locally compact Hausdorff space, and $A$ denotes the $C^{*}$-algebra of bounded Borel functions on $X$ (with pointwise algebra operations, the supremum norm, and complex conjugation $f \mapsto \bar{f}$ as the involution), then the injection $f \mapsto \tilde{f}$ (where $\tilde{f}$ has the same meaning as in Definition 5.4) from $A$ to the enveloping von Neumann algebra $M(X)^{*}$ of $C_{0}(X)$ preserves the *-algebra structure. One way of seeing this is to note that the involution is weak* continuous and multiplication separately weak* continuous in $M(X)^{*}$, then to show that the corresponding statements are true of the operations of $A$ and the topology $\sigma(A, M(X))$, and finally to use the fact that $C_{0}(X)$ is $\sigma(A, M(X))$-dense in $A$ and the restriction of $f \mapsto \tilde{f}$ to $C_{0}(X)$ is a $*$-homomorphism. The separate $\sigma(A, M(X))$ continuity of the pointwise multiplication in $A$ is clear, because

$$
\int f g d \mu=\int f d(g \mu) \text { for } f, g \in A, \mu \in M(X),
$$

where $g \mu$ is the measure $h \mapsto \int(h g) d \mu, h \in C_{0}(X)$. The $\sigma(A, M(X))$ continuity of $f \mapsto \bar{f}$ follows from the identity

$$
\int \bar{f} d \mu=\overline{\int f d \bar{\mu}}, \quad f \in A, \mu \in M(X),
$$

where $\bar{\mu} \in M(X)$ is the measure $h \mapsto\langle\overline{\mu, \bar{h}}\rangle, h \in C_{0}(X)$. This identity can be proved e.g. by interpreting $\mu$ as a set function and writing $\mu=\mu_{1}+i \mu_{2}$ for real measures $\mu_{1}, \mu_{2} \in M(X)$, so that $\bar{\mu}=\mu_{1}-i \mu_{2}$. 
Th e o r e m 5.8. Let $T$ be a locally compact Hausdorff space and $B$ a bounded bimeasure on $\Gamma \times \Gamma$. Let $D$ be any *-subalgebra of the $C^{*}$ algebra of bounded Borel functions on $\Gamma$ such that, for $\mu \in M(\Gamma)$,

$$
\int f d \mu=0 \text { for all } f \in D
$$

only if $\mu=0$. Then the following two conditions are equivalent:

(i) $B(h, \bar{h}) \geq 0$ for all $h \in C_{0}(T)$;

(ii) $B(f, \bar{f}) \geq 0$ for all $f \in D$.

If $\Gamma$ is a locally compact Abelian group with dual group $G$, then e.g.

(i) holds if and only if the function $(s, t) \mapsto B\left((s, \cdot),\left(t^{-1}, \cdot\right)\right)$ on $G \times G$ is a positive-definite kernel.

Proof. In view of the preceding discussion, and the fact that the image

of $D$ (and of $C_{0}(\Gamma)$ ) under the map $f \mapsto \tilde{f}$ is weak* dense in $M(\Gamma)^{*}$, the first assertion is an immediate consequence of Theorem 4.10 and the definition of $B(f, \bar{f})$. The second assertion follows from the first, when $D$ is taken to be the set of all trigonometric polynomials on $\Gamma$.

\section{Applications to stochastic processes}

In the present section, as in the previous one, we apply to a special case the theory presented in Sections 3 and 4. This time we are in the noncommutative situation again, but our operators and their Fourier transforms have their values in a Hilbert space. Throughout, $G$ is an arbitrary locally compact group, and $H$ (unless otherwise specified) is any Hilbert space.

Defin ition 6.1. Every function $\varphi: G \rightarrow H$ is called a stochastic process, and the function $(s, t) \mapsto R(s, t)=(\varphi(s) \mid \varphi(t))$ on $G \times G$ is called its covariance function.

This terminology is of course modelled on the case where $H$ is the (complex) space

$$
L_{0}^{2}(\Omega, \mathscr{A}, P)=\left\{f \in L^{2}(\Omega, \mathscr{A}, P) \mid \int f d P=0\right\}
$$

for some probability space $(\Omega, \mathscr{A}, P)$. Nearly all of the ensuing discussion is independent of this kind of interpretation, but we continue to use the suggestive language of probability theory. Another name for $\varphi: G \rightarrow H$ would be random field.

The next definition recalls a notion introduced (with $\mathbf{R}$ in place of $G$ ) by S. Bochner [2]. We limit our attention to weakly continuous processes. 
D e f in ition 6.2. A bounded weakly continuous stochastic process $\varphi: G \rightarrow H$ is said to be $V$-bounded if the set

is bounded in $H$.

$$
\left\{\int \varphi d \mu \mid \mu \in L^{1}(G),\|\mu\|^{\prime} \leq 1\right\}
$$

Remark 6.3. Since every bounded set in $H$ is relatively weakly compact, Theorem 3.5 shows that a bounded and weakly continuous stochastic process $\varphi: G \rightarrow H$ is $V$-bounded if and only if $\varphi$ is the Fourier transform of some bounded (hence weakly compact) linear operator $\Phi: C^{*}(G) \rightarrow H$. By Theorem 3.2, $\Phi$ is then unique, and $\varphi$ is strongly continuous. By Theorem 3.5 again, in the above definition $L^{1}(G)$ could be replaced by $M(G)$ or $M_{d d}(G)$. This statement concerning $M_{d d}(G)$ has in the case of $H=L_{0}^{2}(\Omega, \mathscr{A}, P)$ the following reformulation:

Theorem 6.4. Let $(\Omega, \mathscr{A}, P)$ be a probability space and $\varphi: G \rightarrow L_{0}^{2}(\Omega, \mathscr{A}, P)$ a weakly continuous stochastic process. Then $\varphi$ is $V$-bounded if and only if there is a constant $C>0$ such that for every finite set $\left\{s_{1}, \ldots, s_{n}\right\} \subset G$ and every vector $z=\left(z_{1}, \ldots, z_{n}\right) \in \mathbf{C}^{n}$ for which $\left\|\sum_{j=1}^{n} z_{j} \delta_{s_{j}}\right\|^{\prime} \leq 1$ we have $E|z \cdot \mathfrak{u}|^{2} \leq C$ where $z \cdot \mathbf{u}$ is the scalar product of $z$ with the random vector (properly speaking, equivalence class) $\mathbf{u}=\left(\varphi\left(s_{1}\right), \ldots, \varphi\left(s_{n}\right)\right)$, and $E$ denotes expectation.

Proof. It suffices to observe that

$$
E|z \cdot \mathbf{u}|^{2}=\int_{\Omega}\left|\sum_{j=1}^{n} z_{j} \varphi\left(s_{j}\right)\right|^{2} d P=\left\|\sum_{j=1}^{n} z_{j} \varphi\left(s_{j}\right)\right\|_{2}^{2} .
$$

The following result generalizes Theorem 3.2.6 in [17, p. 38].

The or e m 6.5. A weakly continuous stochastic process $\varphi: G \rightarrow H$ is $V$-bounded if and only if there is a bounded bilinear form $B: C^{*}(G) \times$ $C^{*}(G) \rightarrow \mathbf{C}$ whose Fourier transform $\hat{B}$ is related to the covariance function $R$ of $\varphi$ by $R(s, t)=\hat{B}\left(s, t^{-1}\right), s, t \in G$. If this is the case, then $B$ is uniquely determined and positive-definite. In fact, $B(x, y)=\left(\Phi x \mid \Phi y^{*}\right)$ for all $x, y \in C^{*}(G)$, where $\Phi: C^{*}(G) \rightarrow H$ is the bounded linear map having $\varphi$ as its Fourier transform.

Proof. Suppose that $\varphi$ is $V$-bounded, and let the bounded operator $\Phi: C^{*}(G) \rightarrow H$ satisfy $\hat{\Phi}=\varphi$. Define $B(x, y)=\left(\Phi x \mid \Phi y^{*}\right)$ for all $x, y \in C^{*}(G)$. The bilinear form $(u, v) \mapsto\left(\Phi^{* *} u \mid \Phi^{* *} v^{*}\right)$ on $C^{*}(G)^{* *} \times$ $C^{*}(G)^{* *}$ is separately weak* continuous and extends $B$, so that it coincides with $\tilde{B}$. In particular, $\hat{B}\left(s, t^{-1}\right)=\left(\Phi^{* *} \omega(s) \mid \Phi^{* *}\left(\omega\left(t^{-1}\right)\right)^{*}\right)=$ $(\varphi(s) \mid \varphi(t))=R(s, t), s, t \in G$. By Theorem 4.5 $B$ is unique. Assume, conversely, that $B: C^{*}(G) \times C^{*}(G) \rightarrow \mathbf{C}$ is a bounded bilinear form such that $\hat{B}\left(s, t^{-1}\right)=R(s, t), s, t \in G$. Then 


$$
\begin{aligned}
\left\|\sum_{j=1}^{n} z_{j} \varphi\left(s_{j}\right)\right\|^{2} & =\sum_{j=1}^{n} \sum_{k=1}^{n} z_{j} \overline{z_{k}}\left(\varphi\left(s_{j}\right) \mid \varphi\left(s_{k}\right)\right) \\
& =\sum_{j=1}^{n} \sum_{k=1}^{n} z_{j} \overline{z_{k}} \tilde{B}\left(\omega\left(s_{j}\right), \omega\left(s_{k}\right)^{*}\right) \\
& =\tilde{B}\left(\sum_{j=1}^{n} z_{j} \omega\left(s_{j}\right),\left(\sum_{k=1}^{n} z_{k} \omega\left(s_{k}\right)\right)^{*}\right) \leq\|B\|\left(\left\|\sum_{j=1}^{n} z_{j} \delta_{s_{j}}\right\|^{\prime}\right)^{2} \\
& \text { for every } \sum_{j=1}^{n} z_{j} \delta_{s_{j}} \in M_{d d}(G)
\end{aligned}
$$

and so $\varphi$ is $V$-bounded (Remark 6.3).

Adapting a definition from [17, p. 29], we introduce the following notion:

Definition 6.6. Let $\varphi: G \rightarrow H$ be weakly continuous and $V$-bounded, and let $\Phi$ and $B$ be as in Theorem 6.5. Then $B$ is called the covariance bilinear form of $\Phi$ (or of $\varphi$ ).

Remark 6.7. Combining Theorem 6.5 with Theorem 4.7 and Corollary 4.8 one obtains several characterizations of the $V$-boundedness of a bounded weakly continuous stochastic process $\varphi: G \rightarrow H$ in terms of its covariance function $R$, analogous to Corolloary 3.2.5 in [17, p. 37]. For example, $\varphi$ is $V$-bounded if and only if the set

$$
\left\{\sum_{j=1}^{m} \sum_{k=1}^{n} z_{j} w_{k} R\left(s_{j}, t_{k}\right)\|\| \sum_{j=1}^{m} z_{j} \delta_{s_{j}}\left\|^{\prime} \leq 1,\right\| \sum_{k=1}^{n} w_{k} \delta_{t_{k}} \|^{\prime} \leq 1\right\}
$$

is bounded.

In the rest of this section we shall discuss the notion of stationarity and the closely related subject of orthogonally scattered operators and bilinear forms.

Definition 6.8. A stochastic process $\varphi: G \rightarrow H$ is said to be (wide-sense) left (resp. right) stationary, if its covariance function $R$ satisfies $R(s, t)=R(u s, u t) \quad(\operatorname{resp} . \quad R(s, t)=R(s u, t u)$ for all $u, s, t \in G$ or, equivalently, if there is a function $\varrho: G \rightarrow \mathbf{C}$ such that $R(s, t)=\varrho\left(t^{-1} s\right)$ (resp. $\left.R(s, t)=\varrho\left(s t^{-1}\right)\right)$ for all $s, t \in G$.

A synonym for "stationary" in this definition would be homogeneous. Both in the case of left and right stationarity the function $\varrho$ is obviously positive-definite. It is a well-known elementary fact that $\varphi$ is strongly continuous if and only if $\varrho$ is continuous, and the weak continuity of $\varphi$ suffices for this to be the case. In the standard Abelian situation, $\varrho$ is utilized in the construction of the spectral representation of a stationary (weakly) continuous $\varphi$ (see e.g. [3, p. 129]). In view of Remark 6.3 and Theorem 6.5, the next theorem transfers that classical representation theorem to the noncommutative case. The first sentence in our theorem generalizes an observation made by $\mathrm{S}$. Bochner [2, p. 21] with $\mathbf{R}$ in place of $G$. 
Th e o r e m 6.9. If $\varphi: G \rightarrow H$ is weakly continuous and left or right stationary, then it is $V$-bounded. A weakly continuous $V$-bounded stochastic process $\varphi: G \rightarrow H$ is left (resp. right) stationary if and only if there is $f \in C^{*}(G)^{*}$ such that the covariance bilinear form $B$ of $\varphi$ satisfies $B(x, y)=$ $\langle f, y x\rangle$ (resp. $B(x, y)=\langle f, x y\rangle)$ for all $x, y \in C^{*}(G)$. Such a functional $f$ is unique and necessarily positive; in fact, $f=T \varrho$, where $\varrho$ is as in Definition 6.8.

Proof. We treat the case of left stationarity; the other case requires only obvious formal changes. Let $\varphi$ be weakly continuous and left stationary, and let $\varrho: G \rightarrow \mathbf{C}$ be the continuous positive-definite function satisfying $(\varphi(s) \mid \varphi(t))=\varrho\left(t^{-1} s\right), s, t \in G$. Define the bounded bilinear form $B: C^{*}(G) \times C^{*}(G) \rightarrow \mathbb{C}$ by $B(x, y)=\langle f, y x\rangle$, where $f$ is the positive functional $T \varrho \in C^{*}(G)^{*}$. Since $(u, v) \mapsto\langle f, v u\rangle$ is separately weak* continuous on $C^{*}(G)^{* *} \times C^{*}(G)^{* *}$ and extends $B$, it is the same as the canonical extension $\tilde{B}$ of $B$, and so we get

$$
\begin{aligned}
\hat{B}\left(s, t^{-1}\right) & =\tilde{B}\left(\omega(s), \omega\left(t^{-1}\right)\right)=\left\langle f, \omega\left(t^{-1} s\right)\right\rangle \\
& =\varrho\left(t^{-1} s\right)=(\varphi(s) \mid \varphi(t)), \quad s, t \in G .
\end{aligned}
$$

Thus $\varphi$ is $V$-bounded by Theorem 6.5. Since $B$ is by definition the covariance bilinear form of $\varphi$, the "only if" part of the second sentence is also proved. Suppose now that $\varphi$ is weakly continuous and $V$-bounded, and $f \in C^{*}(G)^{*}$ satisfies $B(x, y)=\langle f, y x\rangle, x, y \in C^{*}(G)$, where $B$ is the covariance bilinear form of $\varphi$. Define $\varrho=T^{-1} f$. As above, we find that $\hat{B}\left(s, t^{-1}\right)=\varrho\left(t^{-1} s\right)$, i.e. $\varrho\left(t^{-1} s\right)=(\varphi(s) \mid \varphi(t))$ for all $s, t \in G$. Thus $\varphi$ is left stationary, and the expression for $f$ has been established.

If $\Phi: C^{*}(G) \rightarrow H$ is a bounded linear map, it follows from the above theorem that the left or right stationarity of the Fourier transform of $\Phi$ implies that $\Phi$ is "orthogonally scattered" in a sense to be presently specified. Orthogonally scattered operators $\Phi: C^{*}(G) \rightarrow H$ are below given a characterization which shows, in particular, that in the Abelian case this statement can be reversed. In the Abelian case these results are well known in various disguises (see e.g. [3], [13], [15], [16], [19]). Let us begin our study of this theme with a lemma.

$\mathrm{L}$ e $\mathrm{m} \mathrm{ma}$ 6.10. Let $F$ be a topological vector space, which is also an algebra with a separately continuous multiplication $(u, v) \mapsto u v$ and identity element 1. Let $\mathscr{E} \subset F$ be a set of pairwise commuting idempotents such that ef $\in \mathscr{E}$ and $1-e \in \mathscr{E}$ whenever $e, f \in \mathscr{E}$. If $B_{0}: F \times F \rightarrow \mathbf{C}$ is a separately continuous bilinear form such that $B_{0}(e, f)=0$ for all $e$ and $f$ in $\mathscr{E}$ with the property $e f=0$, then $B_{0}(u, v)=B_{0}(u v, 1)$ for all $u$ and $v$ in the closed linear span of $\mathscr{E}$.

Proof. If $e, f \in \mathscr{E}$, then 


$$
\begin{aligned}
B_{0}(e, f) & =B_{0}(e(1-f), f)+B_{0}(e f, f(1-e))+B_{0}(e f, f e) \\
& =B_{0}(e f, e f)=B_{0}(e f, e f)+B_{0}(e f, 1-e f) \\
& =B_{0}(e f, 1) .
\end{aligned}
$$

Our assumptions now imply the assertion at once.

In order to motivate Definition 6.13, we present a theorem on bimeasures. The equivalence of (i), (ii), (iii) and (v) in the theorem has been obtained by different methods (and in the case of a not necessarily bounded bimeasure which obviously requires a modification of (iii); as to this point see, however, Remark 6.12 below) by H. Niemi [18]. We give a proof, because this can be done fairly quickly by using the canonical extension $\tilde{B}$ of a bounded bimeasure $B$. The characteristic function of a set $S$ is denoted by $\xi_{S}$. For the proof of the theorem, recall the discussion preceding Theorem 5.8.

Th e o r e m 6.11. Let $X$ be a locally compact Hausdorff space, and $B$ a bounded bimeasure on $X \times X$. The following five conditions are equivalent: (i) $B(f, g)=0$, if $f, g: X \rightarrow \mathbf{C}$ are continuous functions with disjoint compact supports;

(ii) $B\left(\xi_{K_{1}}, \xi_{K_{2}}\right)=0$, if $K_{1}$ and $K_{2}$ are disjoint compact sets in $X$; (iii) $B\left(\xi_{S_{1}}, \xi_{S_{2}}\right)=0$, if $S_{1}$ and $S_{2}$ are disjoint Borel sets in $X$;

(iv) $\tilde{B}(e, f)=0$, if $e, f \in C_{0}(X)^{* *}$ are projections such that $e f=0$; (v) there is a (necessarily unique) measure $\mu \in M(X)$ such that $B(f, g)=$ $\langle\mu, f g\rangle$ for all $f, g \in C_{0}(X)$.

Proof. Assume first (i). Let $K_{1}$ and $K_{2}$ be disjoint compact subsets of $X$. Recall from Definition 5.4 the notation $\tilde{f}$ for the element of $M(X)^{*}$ determined by a bounded Borel function $f: X \rightarrow \mathbf{C}$. Let $U_{0}$ and $V_{0}$ be disjoint open neighborhoods of $K_{1}$ and $K_{2}$, respectively. When the set $\mathscr{U}$ of the open sets $U$ satisfying $K_{1} \subset U \subset U_{0}$ is directed by the natural order opposite to inclusion, and there is assigned to each $U \in \mathscr{U}$ a continuous function $f_{U}: X \rightarrow[0,1]$ with compact support such that $f(s)=1$ for all $s \in K_{1}$ and $\operatorname{supp}\left(f_{U}\right) \subset U$, it follows from the regularity of the measures in $M(X)$ that the net $\left(\tilde{f}_{U}\right)_{U \in \mathscr{U}}$ converges to $\tilde{\xi}_{K_{1}}$ in $\sigma\left(M(X)^{*}, M(X)\right)$. Similarly, we find a net $\left(g_{V}\right)_{V \in \mathscr{V}}$ of continuous functions whose supports are contained in $V_{0}$, such that the net $\left(\tilde{g}_{V}\right)_{V \in \mathscr{V}}$ converges to $\tilde{\xi}_{K_{2}}$ in $\sigma\left(M(X)^{*}, M(X)\right)$. Since always $\tilde{B}\left(\tilde{f}_{U}, \tilde{g}_{U}\right)=0$, the separate weak* continuity of $\tilde{B}$ now shows that $B\left(\xi_{K_{1}}, \xi_{K_{2}}\right)=\tilde{B}\left(\tilde{\xi}_{K_{1}}, \tilde{\xi}_{K_{2}}\right)=0$. Assume next (ii), and let $S_{1}$ and $S_{2}$ be disjoint Borel sets in $X$. This time we consider the set $\mathscr{K}$ of the compact subsets of $S_{1}$ directed by inclusion, and note that by the regularity of the measures in $M(X)$ the net $\left(\tilde{\xi}_{K}\right)_{K \in \mathscr{K}}$ converges to $\tilde{\xi}_{S_{1}}$ in $\sigma\left(M(X)^{*}, M(X)\right)$. Choosing analogously 
a net convering to $\tilde{\xi}_{S_{2}}$ and using the separate weak* continuity of $\tilde{B}$ we see that $B\left(\xi_{S_{1}}, \xi_{S_{2}}\right)=0$. Assume now (iii). When we take $F=M(X)^{*}$ with the weak* topology, $F, B_{0}=\tilde{B}$, and $\mathscr{E}=\left\{\tilde{\xi}_{S} \mid S \subset X\right.$ is a Borel set $\}$ satisfy the hypotheses of Lemma 6.10 . Since $\mathscr{E}$ separates the points of $M(X)$, its linear span is weak* dense in $M(X)^{*}$, and so $\tilde{B}(u, v)=$ $\tilde{B}(u v, 1)$ for all $u, v \in M(X)^{*}$. We define $\mu \in M(X)$ by $\langle\mu, f\rangle=$ $\tilde{B}(\tilde{f}, 1), f \in C_{0}(X)$. Then $\langle\mu, f g\rangle=\tilde{B}(\tilde{f} \tilde{g}, 1)=\tilde{B}(\tilde{f}, \tilde{g})=B(f, g)$ for all $f, g \in C_{0}(X)$, i.e. (v) holds, the uniqueness of $\mu$ being obvious. Since (iii) implies (v), of course (iv) does so, too. Finally, it is clear that if (v) holds, then $\tilde{B}(u, v)=\langle u v, \mu\rangle$ for all $u, v \in M(X)^{*}$, because $(u, v) \mapsto\langle u v, \mu\rangle$ is separately weak* continuous. Thus (v) implies all the statements (i) through (iv).

Remark 6.12. It is not difficult to extend (the appropriate form of) the above theorem to cover the case of not necessarily bounded bimeasures. Let $\mathscr{K}(X)=\{f: X \rightarrow \mathbf{C} \mid f$ is continuous and $\operatorname{supp}(f)$ is compact $\}$ be regarded in the usual way as the locally convex inductive limit of the Banach spaces $\mathscr{K}(X ; K)=\{f \in \mathscr{K}(X) \mid \operatorname{supp}(f) \subset K\}$ where $K$ ranges over the compact subsets of $X$. For example, if $B: \mathscr{K}(X) \times \mathscr{K}(X) \rightarrow \mathbb{C}$ is a bimeasure (i.e. a continuous bilinear form) which satisfies $B\left(f_{1}, f_{2}\right)=0$ when $f_{1}, f_{2} \in \mathscr{K}(X)$ have disjoint supports, there is a Radon measure $\mu: \mathscr{K}(X) \rightarrow \mathbf{C}$ (i.e. a continuous linear functional) such that $B\left(f_{1}, f_{2}\right)=$ $\mu\left(f_{1} f_{2}\right)$ for all $f_{1}, f_{2} \in \mathscr{K}(X)$. To see this, choose for each $f \in \mathscr{K}(X)$ some $g \in \mathscr{K}(X)$ which has the value 1 on an open set containing $\operatorname{supp}(f)$ and define $\mu(f)=B(f, g)$. From the assumption it follows that this definition does not depend on the choice of $g$. Clearly, $\mu$ is linear and continuous, and Theorem 6.11 can be used to prove that $\mu\left(f_{1} f_{2}\right)=B\left(f_{1}, f_{2}\right)$ for all $f_{1}, f_{2} \in \mathscr{K}(X)$.

In view of Theorem 6.11 , the following definition is consistent with Definition 21 of [18].

Definition 6.13. Let $A$ be a $C^{*}$-algebra and $B: A \times A \rightarrow \mathbf{C}$ a bounded bilinear form. If its canonical extension $B$ satisfies $\tilde{B}(e, f)=0$ for all projections $e, f \in A^{* *}$ such that $e f=0$, then $B$ is said to be orthogonally scattered. A bounded linear map $\Phi: A \rightarrow H$ is said to be orthogonally scattered, if the bilinear form $(x, y) \mapsto\left(\Phi x \mid \Phi y^{*}\right)$ is orthogonally scattered (or, equivalently, if $\left(\Phi^{* *} e \mid \Phi^{* *} f\right)=0$ whenever $e, f \in A^{* *}$ are projections such that $\left.e f=0\right)$.

Th e or e m 6.14. Let $\Phi: C^{*}(G) \rightarrow H$ be a bounded linear map and $B$ its covariance bilinear form, i.e. $B(x, y)=\left(\Phi x \mid \Phi y^{*}\right), x, y \in C^{*}(G)$. Then $\Phi$ is orthogonally scattered if and only if there is $f \in C^{*}(G)^{*}$ such that $\tilde{B}(u, v)=\langle u v, f\rangle$ whenever $u$ and $v$ are commuting normal elements of $C^{*}(G)^{* *}$. If this is the case, then $f$ is a uniquely determined positive func- 
tional, and the continuous positive-definite function $T^{-1} f=\varrho$ on $G$ satisfies

$$
(\hat{\Phi}(s) \mid \hat{\Phi}(t))=\varrho\left(s t^{-1}\right)
$$

whenever $s$ and $t$ are commuting elements of $G$.

Proof. Suppose that $\Phi$ is orthogonally scattered and define $f \in C^{*}(G)^{*}$ by $\langle f, x\rangle=\tilde{B}(\tilde{x}, 1)$ where $\tilde{x} \in C^{*}(G)^{* *}$ is the canonical image of $x \in C^{*}(G)$ and 1 is the identity element of $C^{*}(G)^{* *}$. Then $\langle w, f\rangle=$ $\tilde{B}(w, 1)$ for all $w \in C^{*}(G)^{* *}$, because (the canonical image of) $C^{*}(G)$ is weak* dense in $C^{*}(G)^{* *}$, and $\tilde{B}$ is separately weak* continuous. Let $u$ and $v$ be commuting normal elements of $C^{*}(G)^{* *}$, and denote by $\mathscr{E}$ the set of projections in the (commutative) weak* closed *-subalgebra $M$ of $C^{*}(G)^{* *}$ generated by $u, v$ and 1 . It is a well-known consequence of spectral theory that the linear span of $\mathscr{E}$ is $\sigma\left(C^{*}(G)^{* *}, C^{*}(G)^{*}\right)$-dense (even norm dense, but we do not need this fact) in $M$ (see e.g. [4, p. 3], $\left[20\right.$, p. 26]). Thus we can apply Lemma 6.10 to $F=C^{*}(G)^{* *}$ and $B_{0}=\tilde{B}$, and conclude that $\langle u v, f\rangle=\tilde{B}(u v, 1)=\tilde{B}(u, v)$. It is, conversely, clear that the existence of $f$ with the stated property implies that $\Phi$ is orthogonally scattered. Furthermore, only one linear functional $f \in C^{*}(G)^{*}$ can satisfy $\tilde{B}(u, v)=\langle u v, f\rangle$ for all commuting normal elements of $C^{*}(G)^{* *}$, because we have $w=u+i v$ with selfadjoint $u, \quad v \in C^{*}(G)^{* *}$ for any $w \in C^{*}(G)^{* *}$, so that $\langle w, f\rangle=$ $\langle(u+i v) 1, f\rangle=\tilde{B}(u, 1)+i \tilde{B}(v, 1)=\tilde{B}(w, 1)$. Since every $u \geq 0$ in $C^{*}(G) * *$ has the form $u=v^{2}$ with $v \in C^{*}(G)^{* *}, v \geq 0,\langle u, f\rangle=$ $\tilde{B}(v, v)=\left(\Phi^{* *} v \mid \Phi^{* *} v\right) \geq 0$, i.e. $f$ is positive. Thus $\varrho=T^{-1} f$ is a continuous positive-definite function on $G$. If $s, t \in G$ commute, then $\omega(s)$ and $\omega\left(t^{-1}\right)$ are commuting unitary, hence normal, elements of $C^{*}(G) * *$, and so

$$
\begin{aligned}
\varrho\left(s t^{-1}\right) & =\left\langle f, \omega(s) \omega\left(t^{-1}\right)\right\rangle=\tilde{B}\left(\omega(s), \omega(t)^{*}\right) \\
& =\left(\Phi^{* *} \omega(s) \mid \Phi^{* *} \omega(t)\right)=(\hat{\Phi(s)} \mid \hat{\Phi(t)) .}
\end{aligned}
$$

\section{References}

[1] Akemann, C. A.: The dual space of an operator algebra. - Trans. Amer. Math. Soc. $126,1967,286-302$.

[2] BochNeR, S.: Stationarity, boundedness, almost periodicity of random-valued functions. - Proceedings of the Third Berkeley symposium on mathematical statistics and probability. II, University of California Press, Berkeley-Los Angeles, 1956, 7-27.

[3] CRAMÉR, H., and M. R. LEADBETTTER: Stationary and related stochastic processes. - John Wiley \& Sons, Inc., New York-London-Sydney, 1967. 
[4] Dixmiér, J.: Les algèbres d'opérateurs dans l'espace hilbertien (Algèbres de von Neumann). 2ième éd. - Cahiers scientifiques, Fas. XXV, GauthierVillars, Paris, 1969.

[5] Dixmier, J.: Les $C^{*}$-algèbres et leurs représentations. 2ième éd. - Cahiers scientifiques, Fas. XXIX, Gauthier-Villars, Paris, 1969.

[6] Dunford, N. and J. T. SchWARTZ: Linear operators. Vol. I: General theory. Pure and Appl. Math., vol. 7, Interscience, New York, 1958.

[7] Eberlein, W. F.: Characterizations of Fourier-Stieltjes transforms. - Duke Math. J. 22, 1955, 465-468.

[8] Eymard, P.: L'algèbre de Fourier d'un groupe localement compact. - Bull. Soc. Math. France 92, 1964, $181-236$.

[9] GLICKSBERG, I.: Weak compactness and separate continuity. - Pacific J. Math. 11, 1961, 205-214.

[10] GROTHENDIECK, A.: Sur les applications linéaires faiblement compactes d'espaces du type $C(K)$. - Canad. J. Math. 5, 1953, 129-173.

[11] GROTHENDIECK, A.: Résumé de la théorie métrique des produits tensoriels topologiques. - Bol. Soc. Mat. São Paulo 8 (1953), 1956, $1-79$.

[12] HEwit'T, E., and K. A. Ross: Abstract harmonic analysis. Vol. I. - SpringerVerlag, Berlin - Göttingen - Heidelberg, 1963.

[13] Karhunen, K.: Über lineare Methoden in der Wahrscheinlichkeitsrechnung. Ann. Acad. Sci. Fenn. Ser. A I 37, 1947, 1-79.

[14] KLUVÁNEK, I.: Characterization of Fourier-Stieltjes transforms of vector and operator valued measures. - Czechoslovak Math. J. 17 (92), 1967, $261-277$.

[15] LoÈve, M.: Probability theory. 3rd ed. - D. Van Nostrand Company, Inc., Princeton, N. J.-Toronto-London-Melbourne, 1963.

[16] Masani, P.: Orthogonally scattered measures. - Advances in Math. 2, 1968, $61-117$.

[17] NiEMI, H.: Stochastic processes as Fourier transforms of stochastic measures. Ann. Acad. Sci. Fenn. Ser. A I 591, 1975, 1-47.

[18] NIEMI, H.: On the support of a bimeasure and orthogonally scattered vector measures. - Ann. Acad. Sci. Fenn. Ser. A I 1, 1975, 249-275.

[19] NIEMI, H.: On stationary dilations and the linear prediction of certain stochastic processes. (In preparation.)

[20] Rudin, W.: Fourier analysis on groups. - Interscience Publishers, New YorkLondon, 1962.

[21] SAKAI, S.: $C^{*}$-algebras and $W^{*}$-algebras. - Springer-Verlag, Berlin-Heidelberg-New York, 1971.

[22] SchaEFER, H. H.: Topological vector spaces. - Macmillan, New York, 1966.

[23] Thomas, E.: L'intégration par rapport à une mesure de Radon vectorielle. Ann. Inst. Fourier (Grenoble) 20:2, 1970, 55-191.

\section{University of Helsinki}

Department of Mathematics

SF-00100 Helsinki 10

Finland

Received 22 July 1975 